\title{
Systematic investigation of chemical abundances derived using IR spectra obtained with GIANO*
}

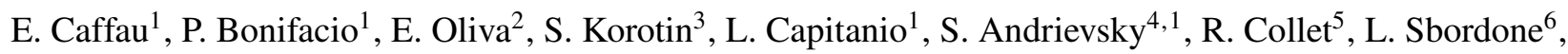 \\ S. Duffau ${ }^{7}$, N. Sanna ${ }^{2}$, A. Tozzi ${ }^{2}$, L. Origlia ${ }^{8}$, N. Ryde ${ }^{9}$, and H.-G. Ludwig ${ }^{10,1}$ \\ ${ }^{1}$ GEPI, Observatoire de Paris, Université PSL, CNRS, 5 Place Jules Janssen, 92190 Meudon, France \\ e-mail: Elisabetta.Caffau@obspm.fr \\ 2 INAF, Osservatorio Astrofisico di Arcetri, Largo E. Fermi 5, 50125 Firenze, Italy \\ ${ }^{3}$ Crimean Astrophysical Observatory, Nauchny 298409, Crimea \\ ${ }^{4}$ Astronomical Observatory, Odessa National University, Shevchenko Park, 65014 Odessa, Ukraine \\ ${ }^{5}$ Stellar Astrophysics Centre, Department of Physics and Astronomy, Aarhus University, 8000 Aarhus C, Denmark \\ ${ }^{6}$ European Southern Observatory, Casilla 19001, Santiago, Chile \\ ${ }^{7}$ Universidad Andrés Bello, Departamento de Ciencias Físicas, Fernandez Concha 700, Las Condes, Santiago, Chile \\ ${ }^{8}$ INAF, Osservatorio Astronomico di Bologna, Via Gobetti 93/340129, Bologna, Italy \\ 9 Lund Observatory, Department of Astronomy and Theoretical Physics, Lund University, Box 43, 22100 Lund, Sweden \\ ${ }^{10}$ Zentrum für Astronomie der Universität Heidelberg, Landessternwarte, Königstuhl 12, 69117 Heidelberg, Germany
}

Received 25 September 2018 / Accepted 26 November 2018

\begin{abstract}
Context. Detailed chemical abundances of Galactic stars are needed in order to improve our knowledge of the formation and evolution of our galaxy, the Milky Way.

Aims. We took advantage of the GIANO archive spectra to select a sample of Galactic disc stars in order to derive their chemical inventory and to compare the abundances we derived from these infrared spectra to the chemical pattern derived from optical spectra. Methods. We analysed high-quality spectra of 40 stars observed with GIANO. We derived the stellar parameters from the photometry and the Gaia data-release 2 (DR2) parallax; the chemical abundances were derived with the code MyGIsFOS. For a subsample of stars we compared the chemical pattern derived from the GIANO spectra with the abundances derived from optical spectra. We derived $\mathrm{P}$ abundances for all 40 stars, increasing the number of Galactic stars for which phosphorus abundance is known.

Results. We could derive abundances of 14 elements, 8 of which are also derived from optical spectra. The comparison of the abundances derived from infrared and optical spectra is very good. The chemical pattern of these stars is the one expected for Galactic disc stars and is in agreement with the results from the literature.

Conclusions. GIANO is providing the astronomical community with an extremely useful instrument, able to produce spectra with high resolution and a wide wavelength range in the infrared.
\end{abstract}

Key words. stars: solar-type - stars: abundances - Galaxy: abundances - Galaxy: disk

\section{Introduction}

The knowledge of the chemical composition of the stars is a necessary ingredient to understand and to model the formation and the evolution of the Milky Way and of the Local-Group galaxies. In order to derive the chemical pattern of a star, astronomers compare the strengths of the atomic and molecular lines in an observed stellar spectrum to theoretical predictions. Typically, spectra with medium/high resolving power $(R>15000)$, a good signal-to-noise ratio $(S / N>50)$, and a wide-enough wavelength range allow deriving precise abundances for several elements. Until a few decades ago, securing stellar spectra was the bottleneck in the chemical investigation of stars. Any single star had to be observed for a reasonable amount of time, depending on its luminosity, the size of the telescope, and the efficiency of the spectrograph, in order to obtain a spectrum with the quality necessary to derive the desired precision in the chemical abundances. For instance, with a four-meter-class telescope, a spectrum with a typical $\mathrm{S} / \mathrm{N}$ of about 50 at $520 \mathrm{~nm}$ is obtained

\footnotetext{
* GIANO programme A31TAC.
}

in one hour for a $V=12 \mathrm{mag}$ star (e.g. HARPS, Mayor et al. 2003) while with an eight-meter-class telescope, a spectrum of the same $\mathrm{S} / \mathrm{N}$ is obtained in one hour for a star 300 times fainter in the $V$ band (e.g. FLAMES, Pasquini et al. 2002). The advent of the multi-object spectrographs (e.g. FLAMES, Pasquini et al. 2002) allowed one to observe on the order of one hundred stars at the same time, considerably increasing the number of stars in the Galaxy for which the chemical composition is known. The Gaia-ESO Survey (Gilmore et al. 2012) is an example of the great efficiency that can be reached by using multi-objects spectrographs. In the near future multi-object spectrographs with a capacity to observe about ten times more stars at the same time are expected (e.g. WEAVE: Dalton et al. 2016 and 4MOST: de Jong et al. 2014).

Before the commissioning of the near-infrared (NIR) highresolution spectrograph GIANO (Origlia et al. 2014) at Telescopio Nazionale Galileo (TNG), the detailed chemical investigations of stars were based mainly on optical spectra. Other IR spectrographs appeared before GIANO, for example CRIRES (Kaeufl et al. 2004). However, their wavelength coverage was small, useful only to add one element on the chemical pattern of 
a star, and did not allow us to perform a systematic investigation of the IR range. GIANO is a cross-dispersed echelle spectrograph, and covers a large wavelength range (950-2450 nm) allowing the abundance of several elements to be derived. The multi-object spectrograph APOGEE (Allende Prieto et al. 2008), with a wavelength range of about $200 \mathrm{~nm}(1510-1700 \mathrm{~nm})$, has been shown to be able to provide detailed chemical composition of Galactic stars. In the future we expect the Multi Object Optical and Near-infrared Spectrograph (MOONS, Cirasuolo et al. 2014), that will be able to observe several hundred objects in three arms with two resolution options. MOONS is a high-multiplicity, multi-object, fibre-fed spectrograph for the ESO VLT. It will be capable of both low $(R \sim 5000)$ and high $(R \sim 20000)$ resolution and will deploy 1000 fibres on the $20^{\prime}$ field of view of the VLT. Its high-resolution $\mathrm{H}$ arm will observe the wavelength range $1521-1641 \mathrm{~nm}$, covered by the orders 47-50 of GIANO.

The present work has two main drivers. On the one hand, we are deeply involved in the preparation of MOONS and wish to be prepared for the millions of spectra it will provide. Therefore a detailed study of the IR range is in order. On the other hand, we are interested in the Galactic evolution of phosphorus, which is still a very little studied element, and of sulphur, both elements being available in the wavelength range of GIANO. Our main goals in this work are: (i) to investigate the IR range and see which elements can be derived and (ii) to derive the abundance of phosphorus. We also wish to provide detailed abundances for several elements for those stars in this sample for which only the abundances of a few elements are known. We took the advantage of the GIANO spectra present in the Italian centre for Astronomical Archives (Molinaro et al. 2018) and we selected a number of dwarf stars (the IR P I lines are contaminated in the spectrum of a giant star) of slightly sub-solar metallicity to super-solar metallicity. For a subsample of stars, SOPHIE (Bouchy \& Sophie Team 2006) spectra of good quality were available, allowing us to compare for some elements the abundances derived from the IR and the optical spectra.

\section{Observations}

Stars were observed with GIANO, the high-resolution $(R \simeq$ $50000)$ IR $(950-2450 \mathrm{~nm})$ spectrometer of the TNG (Origlia et al. 2014). The instrument was designed for direct feeding of light at a dedicated focus of the TNG. In 2012 the spectrometer was provisionally positioned on the rotating building and fed via a pair of IR (ZBLAN) fibres connected to another focal station (Tozzi et al. 2014). In 2016 it was eventually moved to the originally foreseen configuration where it can also be used for simultaneous observations with HARPS-N (Tozzi et al. 2016). The spectra presented here were collected in March 2015, when the spectrometer was still in its provisional configuration and fed via fibres. The raw data were downloaded from the public archive of the TNG. The stars were observed by nodding on fibre, that is, target and sky were taken in pairs and alternatively acquired on the two fibers $(A, B)$ for an optimal subtraction of the detector noise and background. Integration time was $5 \mathrm{~min}$ per A,B position. Dark-subtracted frames of flat (halogen lamp) and wavelength (U-Ne lamp) calibration sources taken at the beginning and/or at the end of each night were used to reduce the data. For each nodding pair we created a two-dimensional (2D) frame by subtracting the raw frames $(\mathrm{A}-\mathrm{B})$ and dividing the difference by the normalised flat. Spectral extraction and wavelength calibration were performed using a physical model of the spectrometer that accurately matches instrumental effects such as variable slit tilt and orders curvature over the echellogram (Oliva et al. 2018). The telluric absorption features were corrected using the spectra of two telluric standards (O-type stars) taken at different airmasses during the same nights. The normalised spectra of the telluric standard taken at low and high airmass values were combined with different weights to match the depth of the telluric lines in the stellar spectra.

In Fig. 1 the $H$-band is shown in the case of the star HD 24040; this range corresponds exactly to the wavelength range that will be observed at high resolution by MOONS.

The SOPHIE spectra have been retrieved from the SOPHIE archive $^{1}$. Several spectra for each star were present in the archive, but only the observations with a corresponding sky spectrum were retained. We subtracted the sky, shifted the spectra at laboratory wavelength and, in the case of several observations, added them together. For one star, HD 76909, we had a single observation.

\section{Stellar parameters}

Within the stars observed by GIANO available in the archive we selected those which had Gaia DR2 (Gaia Collaboration 2018a,b) parallax as well as 2MASS (Cutri et al. 2003) and Tycho (ESA 1997) photometry. To derive effective temperatures we used the colour-temperature calibrations of González Hernández \& Bonifacio (2009) that are based on temperatures derived through the IR flux method for a sample of calibrators. To do so we used the colour transformations given by Eqs. (1.3.20) and (1.3.26) in the HIPPARCOS and Tycho catalogues (ESA 1997) ${ }^{2}$ to obtain $V$ and $(B-V)$ in the Johnson system. All the stars are fairly near and little reddened, nevertheless we derived the reddening for each star from the reddening maps of Capitanio et al. (2017) and corrected the colours for reddening. We then applied the equations given in Table 5 of González Hernández \& Bonifacio (2009) to derive effective temperatures $\left(T_{\text {eff }}\right)$ from $(B-V),(V-J),(V-H),(V-J)$ and $(V-K)$ that were averaged among the different calibrations. The colours involving the 2MASS photometry were retained only if the corresponding quality flag was A. This implies that not all stars have the same number of colour determinations. In Table A.1, $T_{\text {eff }}$ is provided with an uncertainty which is the scatter among the temperatures derived by the five calibrations. For one star, HD 190360, $T_{\text {eff }}$ is derived from one single calibration (that using the $B-V$ colour), and we assumed $250 \mathrm{~K}$ as uncertainty in the temperature; for two stars, HD 28005 and HD 34575 , four calibrations were used (those based on colours $B-V$, $V-J, V-K$ and $J-K$ ), and for all the other stars the five calibrations were used. The effective temperature of this sample of stars spans from 5006 to $6292 \mathrm{~K}$, with $\left\langle T_{\text {eff }}\right\rangle=(5727 \pm 245) \mathrm{K}$.

For each calibration used to derive the effective temperature, we derived the corresponding surface gravity from the Stefan-Boltzmann relation using the distances from the parallaxes provided by Gaia DR2 and the stellar absolute luminosity, derived from the Gaia $G$ band and a bolometric correction based on three-dimensional (3D) model atmospheres (Bonifacio et al. 2018). We assumed the mass of each star according to its temperature, by looking at the isochrones. The uncertainty in the gravity is the scatter among the values we derived, and this value is on average below 0.05 and for all stars below 0.1 . The gravities we derived spanned from 3.53 to 4.56 (cgs), with $\langle\log g\rangle=$ $4.16 \pm 0.25$ (see Table A.1).

\footnotetext{
http://atlas.obs-hp. fr/sophie/

$2 V_{J}=V_{T}-0.090 \times(B-V)_{T} ; z=B_{T}-V_{T}-0.90 ;(B-V)_{J}=$ $\left(B_{T}-V_{T}\right)-0.115-0.229 z+0.043 z^{3}$.
} 


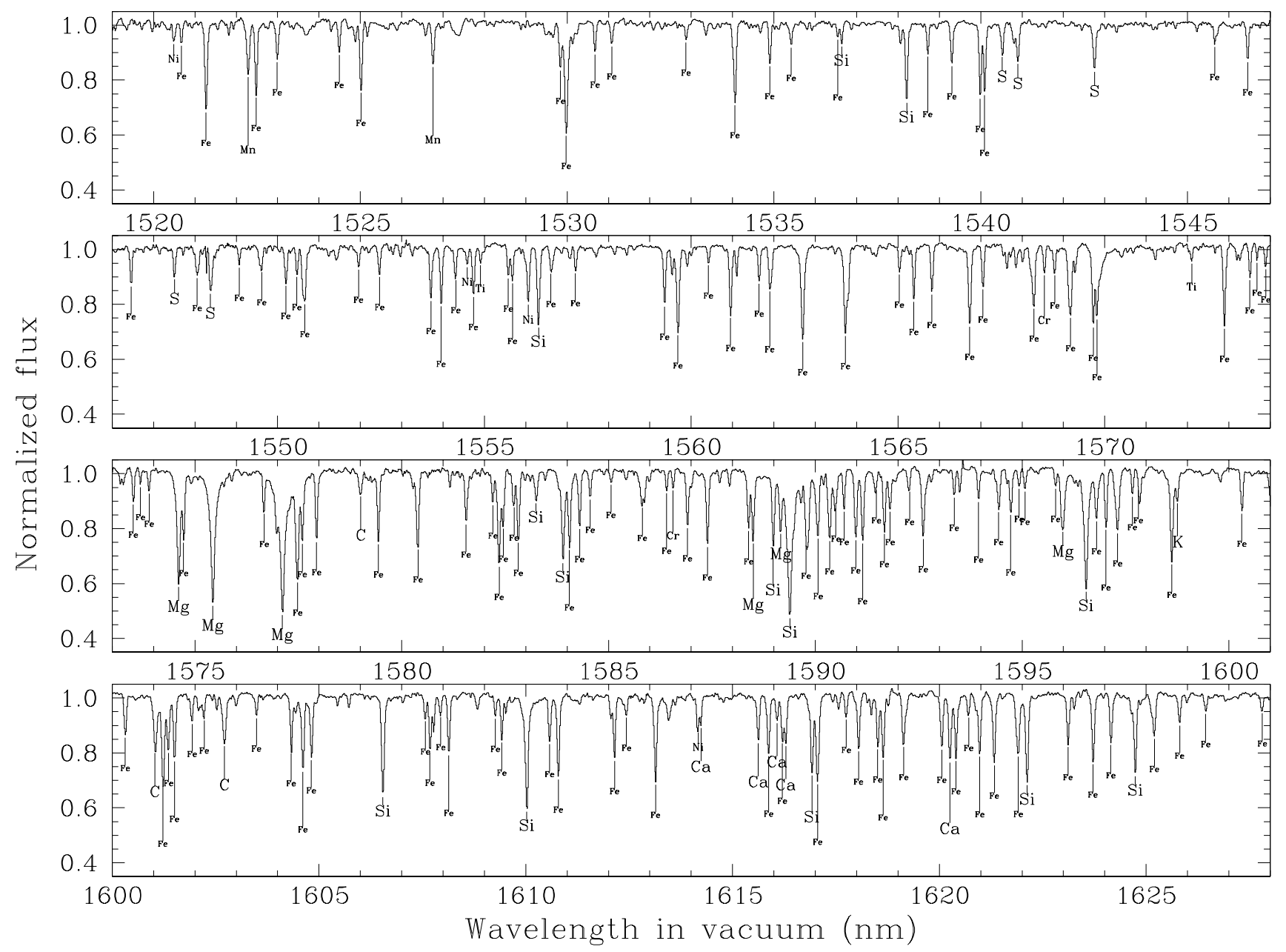

Fig. 1. The $H$-band in the case of HD 24040 (from top to bottom panels orders from 50 to 47).

The stars we are analysing have a metallicity from slightly sub-solar to super-solar, meaning that the lines are strong and several lines are blended. Also, to derive the micro-turbulence in the IR range is not trivial since the lines we can detect are stronger than in the optical range. We then fixed the value for the microturbulence at $1.0 \mathrm{~km} \mathrm{~s}^{-1}$, which is a good value for this sample of dwarf stars. Also, for the Sun a microturbulence at $1.0 \mathrm{~km} \mathrm{~s}^{-1}$ is the preferred value (Steffen et al. 2013).

The chemical analysis has been done with the pipeline MyGIsFOS (Sbordone et al. 2014), in the complete wavelength range provided by GIANO, except the ranges that are too heavily contaminated by telluric absorption. This choice was also motivated by two facts: (i) the GIANO archive is providing more and more stars that can be analysed; (ii) the future MOONS-GTO as well as the future MOONS observations will imply several thousand spectra each night. The numbers of stars to analyse is too large to reasonably allow a by-hand analysis. To analyse the present sample of stars, MyGIsFOS operated at fixed $T_{\text {eff }}$ and $\log g$ and derived, from selected atomic lines, the chemical abundances by comparing the observed spectrum to a grid of synthetic spectra. The theoretical synthesis was computed by SYNTHE (see Kurucz 2005; Sbordone et al. 2004) from a grid of ATLAS 12 models (Kurucz 2005) with a step of 0.25 dex in metallicity. The atomic parameters of the lines are from the most recent line-list provided by Kurucz on his site ${ }^{3}$; in the $H$ band we used the atomic data provided by Shetrone et al. (2015). The stars we

\footnotetext{
3 http://kurucz.harvard.edu/linelists.html
}

analysed all had metallicities from slightly sub-solar to supersolar, and from Fe I lines we derived for the sample of stars: $\langle[\mathrm{Fe} / \mathrm{H}]\rangle=+0.10 \pm 0.11$; for all the stars $-0.14 \leq[\mathrm{Fe} / \mathrm{H}] \leq$ +0.33 . Similar results were derived using Fe II lines, $-0.07 \leq$ $[\mathrm{Fe} / \mathrm{H}] \leq+0.40$, with an average difference of $[\mathrm{Fe} / \mathrm{H}]$ from $\mathrm{Fe} \mathrm{I}$ and Fe II lines smaller than 0.01 dex, the largest one being just smaller than 0.10 dex. The difference of $[\mathrm{Fe} / \mathrm{H}]$ from $\mathrm{Fe} I$ and $\mathrm{Fe}$ II lines is in any case smaller than the uncertainties (see Table A.1).

\section{Comparison optical-IR}

For nine stars (see Table 1), we had optical SOPHIE spectra for which one fibre was on the sky. We could then subtract the sky and compare the analysis of GIANO and SOPHIE for the elements in common.

Eight elements are in common in the two analyses and the agreement is generally very good.

- The agreement in the Fe abundance derived from optical and IR spectra is good: $\left\langle A(\mathrm{Fe} \mathrm{I})_{\mathrm{IR}}-A(\mathrm{Fe} \mathrm{I})_{\mathrm{opt}}\right\rangle=-0.02 \pm 0.02$ (see Fig. 2) and $\left\langle A(\mathrm{Fe} \text { II })_{\mathrm{IR}}-A(\mathrm{Fe} \text { II })_{\text {opt }}\right\rangle=0.03 \pm 0.09$. For one star in particular (HD 76909), the difference on $A(\mathrm{Fe}$ II) from optical and IR spectra is large $(0.18 \mathrm{dex})$ but still within uncertainties; in fact it is the optical spectrum that shows a larger disagreement in the $A(\mathrm{Fe})$ from $\mathrm{Fe} I$ and $\mathrm{Fe}$ II lines and the line-to-line scatter from the Fe II lines is the largest among the optical spectra. 

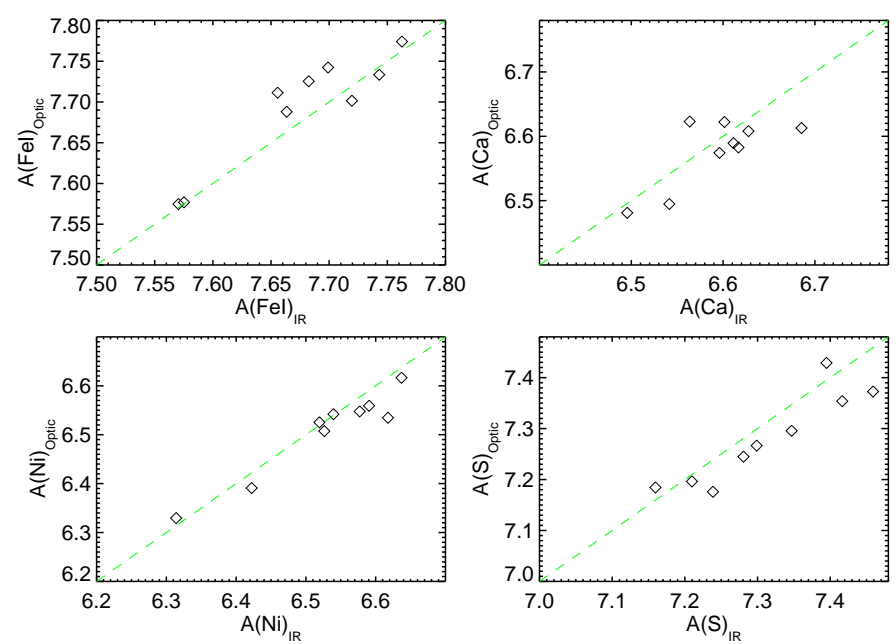

Fig. 2. Comparison of the abundances derived from IR and optical spectra.

Table 1. Sample of stars with optical and IR spectra.

\begin{tabular}{lrrrrr}
\hline \hline \multirow{2}{*}{ Star } & \multirow{2}{*}{$T_{\text {eff }}$} & \multirow{2}{*}{$\log g$} & \multirow{2}{c}{$\xi$} & \multicolumn{2}{c}{$[\mathrm{Fe} / \mathrm{H}]$} \\
& $\mathrm{K}$ & $(\mathrm{cgs})$ & $\mathrm{km} \mathrm{s}^{-1}$ & GIANO & Optical \\
\hline HD 34575 & 5582 & 4.22 & 1.0 & 0.18 & 0.22 \\
HD 67346 & 5953 & 3.78 & 1.0 & 0.14 & 0.19 \\
HD 69056 & 5637 & 4.28 & 1.0 & 0.05 & 0.05 \\
HD 69960 & 5655 & 3.99 & 1.0 & 0.22 & 0.21 \\
HD 73933 & 6143 & 4.24 & 1.0 & 0.06 & 0.06 \\
HD 76909 & 5655 & 4.17 & 1.0 & 0.24 & 0.25 \\
HD 90681 & 5950 & 4.26 & 1.0 & 0.16 & 0.21 \\
HD 97645 & 6127 & 4.10 & 1.0 & 0.14 & 0.17 \\
HD 108942 & 5882 & 4.27 & 1.0 & 0.20 & 0.18 \\
\hline
\end{tabular}

- There is a systematic difference in $A(\mathrm{Na})$ from IR and optical spectra: $\left\langle A(\mathrm{Na})_{\mathrm{IR}}-A(\mathrm{Na})_{\mathrm{opt}}\right\rangle=-0.23 \pm 0.14$. From the IR and optical spectra we have $\langle[\mathrm{Na} / \mathrm{Fe}]\rangle=-0.11 \pm 0.09$ and $\langle[\mathrm{Na} / \mathrm{Fe}]\rangle=+0.11 \pm 0.08$, respectively. This difference could be due to NLTE effects; in fact the IR lines form close to LTE, but this is not true for the optical lines, which are sensitive to NLTE.

- The agreement in $A(\mathrm{Al})$ is really good; in the case of IR spectra, in seven cases out of nine, $A(\mathrm{Al})$ is derived from one single line and in the other two stars from two lines: $\left\langle A(\mathrm{Al})_{\mathrm{IR}}-A(\mathrm{Al})_{\text {opt }}\right\rangle=+0.07 \pm 0.06$.

- For $A(\mathrm{Si})$ from $\mathrm{Si}$ I lines, the agreement of IR and optical spectra is good, well within the uncertainties: $\left\langle A(\mathrm{Si})_{\mathrm{IR}}-\right.$ $\left.A(\mathrm{Si})_{\text {opt }}\right\rangle=-0.08 \pm 0.04$.

- Also the agreement for $A(\mathrm{~S})$ is extremely good: $\left\langle A(\mathrm{~S})_{\mathrm{IR}}-\right.$ $\left.A(\mathrm{~S})_{\text {opt }}\right\rangle=+0.03 \pm 0.04$ (see Fig. 2).

- For $A(\mathrm{Ca})$ from $\mathrm{Ca} I$ lines the agreement is also perfect: $\left\langle A(\mathrm{Ca})_{\mathrm{IR}}-A(\mathrm{Ca})_{\text {opt }}\right\rangle=+0.02 \pm 0.04$ (see Fig. 2).

- The Ti abundance, derived from Ti I and Ti II lines is very close from IR and optical spectra: $\left\langle A(\mathrm{Ti} \mathrm{I})_{\mathrm{IR}}-A(\mathrm{Ti} \mathrm{I})_{\mathrm{opt}}\right\rangle=$ $-0.05 \pm 0.05$ and $\left\langle A(\mathrm{Ti} \text { II })_{\mathrm{IR}}-A(\mathrm{Ti} \text { II })_{\mathrm{opt}}\right\rangle=0.08 \pm 0.05$.

- For Ni we also find a good agreement between IR and optical: $\left\langle A(\mathrm{Ni})_{\mathrm{IR}}-A(\mathrm{Ni})_{\text {opt }}\right\rangle=+0.02 \pm 0.03$ (see Fig. 2).

A summary on the comparison of the abundances derived from

IR and optical spectra is presented in Table 2.

Previously published investigations exist for all nine stars from Table 1. Three of the papers that contain parameters for
Table 2. Comparison of abundances from IR and optical spectra.

\begin{tabular}{lrr}
\hline \hline Ion & IR-optical & rms \\
\hline Na I & -0.23 & 0.14 \\
Al I & +0.07 & 0.06 \\
Si I & -0.08 & 0.04 \\
S I & +0.03 & 0.04 \\
Ca I & +0.02 & 0.04 \\
Ti I & -0.05 & 0.05 \\
Ti II & +0.08 & 0.05 \\
Fe I & -0.02 & 0.02 \\
Fe II & +0.03 & 0.09 \\
Ni I & +0.02 & 0.03 \\
\hline
\end{tabular}

some of these stars are based on spectra (Valenti \& Fischer 2005; Petigura \& Marcy 2011; Brewer et al. 2016) and two are based on photometry (Masana et al. 2006; Casagrande et al. 2011). All the spectra used in Valenti \& Fischer (2005), Petigura \& Marcy (2011), and Brewer et al. (2016) are optical; all have been collected in the course of planet search campaigns and have S/N ratios exceeding 100 . Valenti \& Fischer (2005) used three different spectrographs (HIRES at Keck, Hamilton at Lick, and UCLES at the AAT), mostly with a resolution of $R \sim 70000$, comparable to our SOPHIE spectra. Also, Petigura \& Marcy (2011) used HIRES spectra, but at a somewhat lower resolution, $R \sim 50000$. Finally Brewer et al. (2016) used HIRES at Keck, and used several resolutions in the range $57000 \leq R \lessgtr 78000$. In the following we discuss abundances in terms of $A(X)=$ $\log (X / H)+12$.

\section{1. $H D 34575$}

For this star we derived $T_{\text {eff }}=5582 \mathrm{~K}$, in very good agreement with $5529 \mathrm{~K}$ derived by Masana et al. (2006) and $5546 \mathrm{~K}$ derived by Casagrande et al. (2011). The analysis by Brewer et al. (2016) is in very good agreement with our investigation; they derive very close stellar parameters $\left(T_{\text {eff }}=5551 \mathrm{~K}, \log g=4.25\right)$ and also the abundances $(\mathrm{C}, \mathrm{Na}, \mathrm{Al}, \mathrm{Si}, \mathrm{Ca}, \mathrm{Ti}, \mathrm{Fe})$ are in agreement with our findings, well within the uncertainties. Valenti \& Fischer (2005) derived a close effective temperature, $5651 \mathrm{~K}$, and a gravity 0.23 dex larger than our adopted value. The abundances they derive ( $\mathrm{Na}, \mathrm{Si}, \mathrm{Ti}, \mathrm{Fe}, \mathrm{Ni}$ ) are mostly slightly larger, but well compatible within the uncertainties. With the stellar parameters from Valenti \& Fischer (2005), Petigura \& Marcy (2011) derived a $\mathrm{C}$ abundance for this star that is compatible with our value, but larger, as in the abundances from Valenti \& Fischer (2005).

\section{2. $H D 67346$}

The temperature we derive for this star of $5953 \mathrm{~K}$ is in close agreement with $T_{\text {eff }}=5941 \mathrm{~K}$ from Masana et al. (2006), but $200 \mathrm{~K}$ cooler than Casagrande et al. (2011; $\left.T_{\text {eff }}=6157 \mathrm{~K}\right)$. With a $T_{\text {eff }}$ that is $140 \mathrm{~K}$ hotter and a gravity of $0.4 \mathrm{dex}$ higher, Petigura \& Marcy (2011) derived higher C and Ni abundances.

\section{3. $H D 69056$}

The effective temperature we derive $\left(T_{\text {eff }}=5637 \mathrm{~K}\right)$ for this star is in very good agreement with the values from Masana et al. (2006) and Casagrande et al. (2011; $T_{\text {eff }}$ of 5598 and $5635 \mathrm{~K}$, respectively). Petigura \& Marcy (2011) used $T_{\text {eff }}=5490 \mathrm{~K}$ and 
$\log g$ of 4.27 and derived $\mathrm{C}$ and $\mathrm{Ni}$ abundances in agreement with our results.

\section{4. $H D 69960$}

Our $T_{\text {eff }}=5655 \mathrm{~K}$ is in extremely good agreement with $T_{\text {eff }}=5625 \mathrm{~K}$ by Casagrande et al. (2011) and also in agreement with the cooler value of Masana et al. $\left(2006 ; T_{\text {eff }}=5572 \mathrm{~K}\right)$. The gravity we derived is of 3.99. Brewer et al. (2016) with very similar stellar parameters, derived abundances $(\mathrm{C}, \mathrm{Na}, \mathrm{Mg}$, $\mathrm{Al}, \mathrm{Si}, \mathrm{Ca}, \mathrm{Ti} \mathrm{Cr}, \mathrm{Fe}, \mathrm{Ni}$ ) that are in general good agreement within uncertainties, with our results. With stellar parameters of $T_{\text {eff }}=5690 \mathrm{~K}$ and $\log g=4.21$, Petigura \& Marcy (2011) derive $A(\mathrm{C})=8.76$, consistent with our value within uncertainties.

We are, by and large, in agreement with Chen et al. (2008), in spite of their effective temperature being about $200 \mathrm{~K}$ cooler.

\section{5. $H D 73933$}

The effective temperature we derive $(6243 \mathrm{~K})$ is about $100 \mathrm{~K}$ hotter than the values from Casagrande et al. (2011) and Masana et al. (2006). Petigura \& Marcy (2011), with an effective temperature of $6076 \mathrm{~K}$ and $\log g=4.39$, derived a $\mathrm{C}$ abundance in agreement with our value, within uncertainties

\section{6. $H D 76909$}

The $T_{\text {eff }}=5655 \mathrm{~K}$ we adopt is in very good agreement with Masana et al. (2006). Valenti \& Fischer (2005) adopted an effective temperature about $100 \mathrm{~K}$ higher, a gravity 0.2 dex higher, and derive a metallicity about 0.1 dex higher. Our results are in very good agreement with those of Brewer et al. (2016) both for the stellar parameters and the detailed abundances $(\mathrm{C}, \mathrm{Mg}, \mathrm{Al}, \mathrm{Si}$, $\mathrm{Ca}, \mathrm{Ti}, \mathrm{Cr}, \mathrm{Fe}, \mathrm{Ni}$ ). Only our $\mathrm{Na}$ abundance is definitely larger. Petigura \& Marcy (2011) adopted parameters compatible with ours ( $T_{\text {eff }}=5740 \mathrm{~K}$ and $\left.\log g=4.39\right)$ to derive a $\mathrm{C}$ abundance in perfect agreement with our value.

We remind the reader that we used only one SOPHIE spectrum for the optical analysis, the only one that has the sky.

\section{7. $H D 90681$}

For this star we adopted $T_{\text {eff }}=5950 \mathrm{~K}$ and $\log g=4.26$. With very close stellar parameters $\left(T_{\text {eff }}=5995 \mathrm{~K}\right.$ and $\left.\log g=4.49\right)$, Petigura \& Marcy (2011) derived the same C abundance.

\section{8. $H D 97645$}

We adopted $T_{\text {eff }}=6127 \mathrm{~K}$ and $\log g=4.10$. With consistent stellar parameters $\left(T_{\text {eff }}=6171 \mathrm{~K}\right.$ and $\left.\log g=4.32\right)$, Petigura \& Marcy $(2011)$ derived a $\mathrm{C}$ abundance $(A(\mathrm{C})=8.51)$ very close to our value of $A(\mathrm{C})=8.48$.

\section{9. $H D 108942$}

We adopted stellar parameters $\left(T_{\text {eff }}=5882 \mathrm{~K}\right.$ and $\left.\log g=4.27\right)$ in good agreement with the literature (Masana et al. 2006; Casagrande et al. 2011; Ramírez et al. 2012). Petigura \& Marcy (2011), adopting a slightly smaller $T_{\text {eff }}=5795 \mathrm{~K}$ and higher $\log g=4.32$, derived abundances ( $\mathrm{C}, \mathrm{Na}, \mathrm{Mg}, \mathrm{Al}, \mathrm{Si}, \mathrm{Ti}, \mathrm{Cr}$, $\mathrm{Fe}, \mathrm{Ni}$ ) in agreement, within uncertainties, with our values.

\subsection{Granulation effects}

We investigated whether the effects of granulation on abundance determinations are similar at optical and IR wavelengths.
In order to assess this, we synthesised a number of fictitious Fe I and Fe II lines of varying strength with excitation potentials between 3 and $5 \mathrm{eV}$ at $500 \mathrm{~nm}$ (optical), $850 \mathrm{~nm}$ (NIR), and $1600 \mathrm{~nm}$ (IR) using both 1D and 3D model atmospheres of solar-metallicity $([\mathrm{Fe} / \mathrm{H}]=+0.0)$ for stars with physical parameters representative of the present sample: $T_{\text {eff }}$ in the range $5000-6500 \mathrm{~K}$ and $\log g=4.0\left(\mathrm{~cm} \mathrm{~s}^{-2}\right)$ plus the Sun $\left(T_{\text {eff }}=5771 \mathrm{~K}\right.$ and $\left.\log g=4.44\right)$. The 3D hydrodynamic and corresponding stationary $1 \mathrm{D}$ hydrostatic models were adopted from the STAGGER-GRID (Magic et al. 2013). We used these models in combination with the SCATE code (Hayek et al. 2011) to compute spectral line profiles under the assumption of local thermodynamic equilibrium (LTE). For the calculations with 1D models, we adopted a micro-turbulent parameter of $\xi=1.0 \mathrm{~km} \mathrm{~s}^{-1}$. We derived the 3D-1D abundance corrections for the fictitious lines with varying strength by comparing the 3D and 1D Fe abundances required to match a given equivalent width $(\mathrm{EW})$ value. We then quantified the differential granulation effects between different spectral regions by computing the 3D-1D abundance corrections of lines from the same ion at two different wavelengths, for example, optical and IR (but with identical excitation potential and identical normalised equivalent width $\mathrm{EW} / \lambda$ ) and analysing the difference. We find the differential granulation effects for unsaturated Fe II lines at optical and IR wavelengths to be generally small, ranging from about $0.0 \mathrm{dex}$ at $T_{\text {eff }}=5500 \mathrm{~K}$ to about $0.06 \mathrm{dex}$ at $T_{\text {eff }}=6500 \mathrm{~K}$. Differential granulation effects for Fe I lines are typically $0.01-0.02$ dex larger, but following a similar trend with effective temperature. For comparison, differential 3D-1D abundance corrections between NIR and IR lines show a flatter trend with values in the range $0.00-0.05$ dex. Results are summarised in Fig. 3. Calculations performed with models from the CIFIST grid (Ludwig et al. 2009) show similar behaviour for the differential granulation effects (see also Caffau \& Ludwig 2007).

\section{Detailed chemical investigation of the full sample}

We were able to derive the abundance of 14 elements (C, $\mathrm{Na}, \mathrm{Mg}$, $\mathrm{Al}, \mathrm{Si}, \mathrm{P}, \mathrm{S}, \mathrm{K}, \mathrm{Ca}, \mathrm{Ti}, \mathrm{Cr}, \mathrm{Fe}, \mathrm{Ni}, \mathrm{Sr}$ ) from the GIANO spectra. These are reported in Tables A.1-A.5. Some lines of Mn I are present in the range of GIANO $(1297,1328,1329,1521 \mathrm{~nm})$, but the abundance we derive is not trustworthy, probably due to departure from LTE or granulation effects.

\subsection{Phosphorus}

Phosphorus has a single stable isotope ${ }^{31} \mathrm{P}$, which is probably produced via neutron capture and the most likely site where it is produced is in the oxygen and neon burning shells during late stages of massive stars (see e.g. Caffau et al. 2011).

With this analysis, we added 40 points in the $[\mathrm{P} / \mathrm{Fe}]$ versus $[\mathrm{Fe} / \mathrm{H}]$ diagram. The $\mathrm{P}$ I lines we looked at are two of the lines of Mult. 1 at 1053.24 and $1058.44 \mathrm{~nm}(\log$ gf of 0.20 and 0.48 , respectively), which have already been used by Caffau et al. $(2011,2016)$, and a line of Mult. 4 at $1008.70 \mathrm{~nm}$ (with $\log g f=0.130)$. The two lines of Mult. 1 are shown in Fig. 4 in the case of HD 24040. The P abundance we derive from the $1008.70 \mathrm{~nm}$ line is on average larger ( 0.06 and $0.15 \mathrm{dex})$ than the one derived from the other two lines. This difference is not particularly large, the difference between the two lines of Mult. 1 being 0.09 dex.

In Fig. 5, we compare these results with previous analyses. In particular, Caffau et al. $(2011,2016)$ claimed a behaviour 


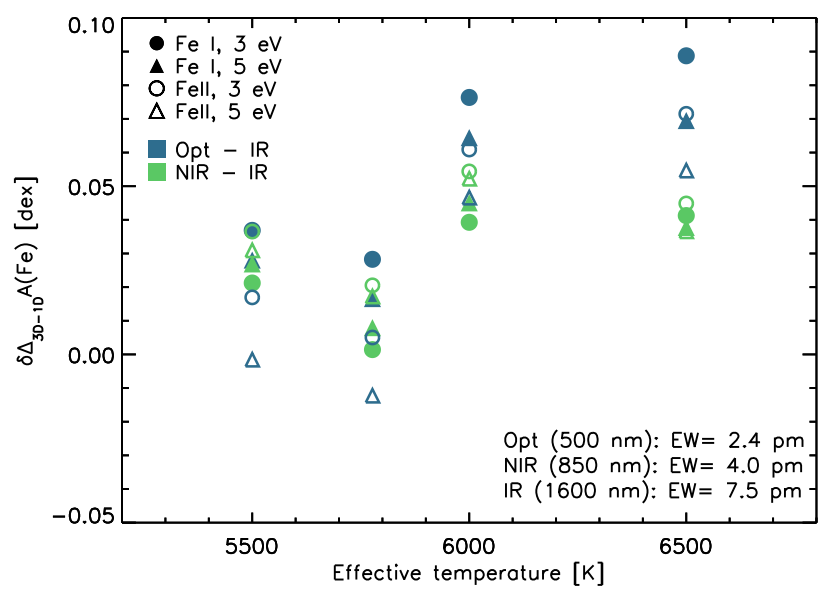

Fig. 3. Differential granulation effects, i.e. differential 3D-1D abundance corrections $\delta \Delta_{3 \mathrm{D}-1 \mathrm{D}} A(\mathrm{Fe})$, between optical and IR and between NIR and IR wavelengths derived from moderately high-excitation unsaturated $\mathrm{Fe}$ lines for dwarf stars as a function of effective temperature. Filled and open symbols refer to Fe I and Fe II lines, respectively. Circles and triangles represent lines with a lower-level excitation potential of 3 and $5 \mathrm{eV}$, respectively. Blue (green) symbols indicate differential corrections between optical and IR (NIR and IR) wavelengths. Abundance corrections are computed for fictitious lines with the same normalised equivalent width, $\mathrm{EW} / \lambda$, and corresponding to a value of $w_{\lambda}=4 \mathrm{pm}$ at $\lambda=850 \mathrm{~nm}$.

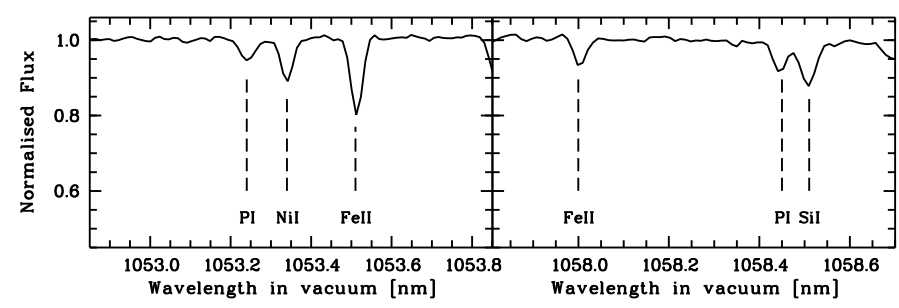

Fig. 4. The two lines of P I belonging to Mult. 1 for the star HD 24040.

of $\mathrm{P}$ similar to an $\alpha$-element, such as S. Unfortunately, the metallicity of this sample of stars is too high to be able to confirm this finding.

\subsection{Sulphur}

Sulphur is an $\alpha$ element produced by type-II supernovae. The fact that sulphur is a relatively volatile element, preventing it from being locked into dust grains in the interstellar medium, makes it a perfect element to compare $\alpha$ abundances in stars and in blue compact galaxies and damped Ly $\alpha$ systems. Details on $\mathrm{S}$ and an exhaustive summary of the results in the literature can be found in Duffau et al. (2017).

To derive $A(\mathrm{~S})$, we investigated the three $\mathrm{S}$ I lines of Mult. 3 at $1045 \mathrm{~nm}$ as we did in Caffau et al. (2016). We also looked at the triplet at $1541 \mathrm{~nm}$, one single line in the multiplet at $1547 \mathrm{~nm}$ and the multiplet at $2261 \mathrm{~nm}$ (see Fig. 6 in the case of HD 24040). This multiplet is in a wavelength range contaminated by telluric absorptions that have to be removed when it is analysed.

We can compare the $\mathrm{S}$ abundance derived here with the metal-rich sample analysed by Ecuvillon et al. (2004) and with our previous investigations on sulphur. In Fig. 7, we added the sample analysed here to the metal-rich range of the plot we showed in Duffau et al. (2017); the agreement with the previous investigations is very good. The fact that $[\mathrm{S} / \mathrm{Fe}]$ analysed here is on average slightly higher than the result by

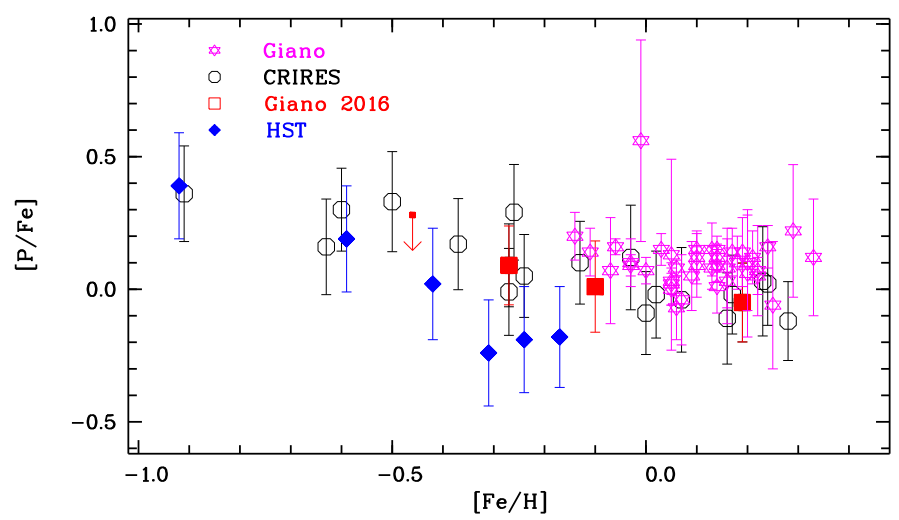

Fig. 5. $[\mathrm{P} / \mathrm{Fe}]$ vs. $[\mathrm{Fe} / \mathrm{H}]$ is shown for the programme stars (pink stars). The four stars analysed by Caffau et al. (2016) are the solid red squares, the results from Caffau et al. (2011) are the black open circles, and the most metal-poor sample is from Roederer et al. (2014; solid blue diamonds).

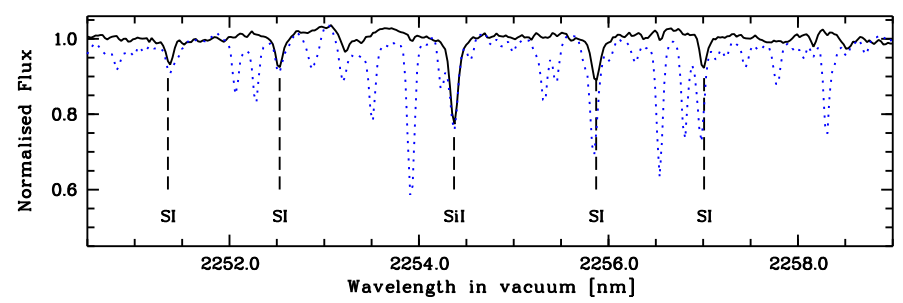

Fig. 6. Four of the S I lines belonging to a multiplet (solid black) in the case of the star HD 24040. The spectrum (dotted blue) before the correction of the telluric lines is overplotted.

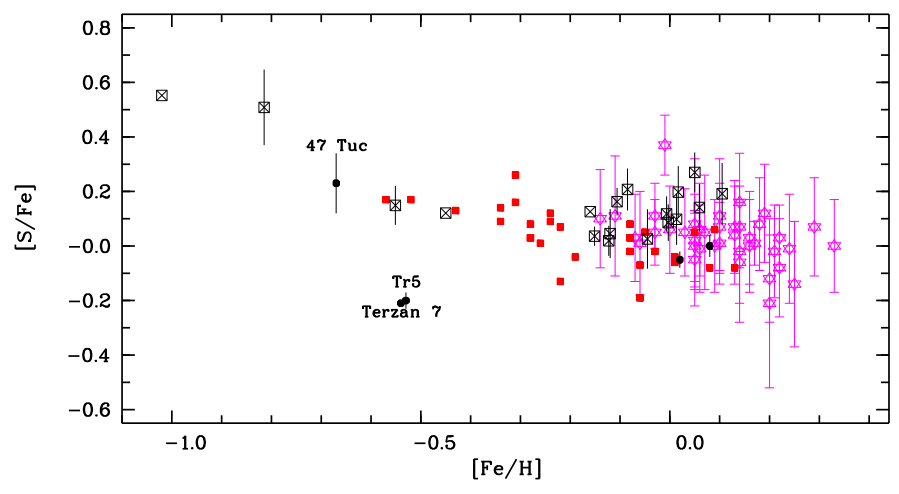

Fig. 7. An update of the plot shown by Duffau et al. (2017) in its metalrich range, for $[\mathrm{S} / \mathrm{Fe}]$ vs. $[\mathrm{Fe} / \mathrm{H}]$. The program stars are shown as pink stars. The red filled squares are from Ecuvillon et al. (2004).

Ecuvillon et al. (2004) could be explained by the fact that among the $\mathrm{S}$ I lines that we adopt to derive the $\mathrm{S}$ abundance, the lines of Mult. 3 at $1045 \mathrm{~nm}$ are affected by NLTE, which would slightly decrease $A(\mathrm{~S})$, while Ecuvillon et al. (2004) analysed the lines of Mult. 8 formed close to the LTE condition.

\subsection{The other elements}

We were able to find several atomic lines of $\mathrm{C} I$ in the GIANO wavelength range. This is extremely important because nitrogen can be derived from $\mathrm{CN}$ molecules once the $\mathrm{C}$ abundance is known. $[\mathrm{C} / \mathrm{Fe}]$ is consistent with Galactic stars of slightly subsolar to super-solar metallicity $([\mathrm{C} / \mathrm{Fe}]=-0.04 \pm 0.08)$ with just one star, HD 190360, with $[\mathrm{C} / \mathrm{Fe}]>+0.3$. 


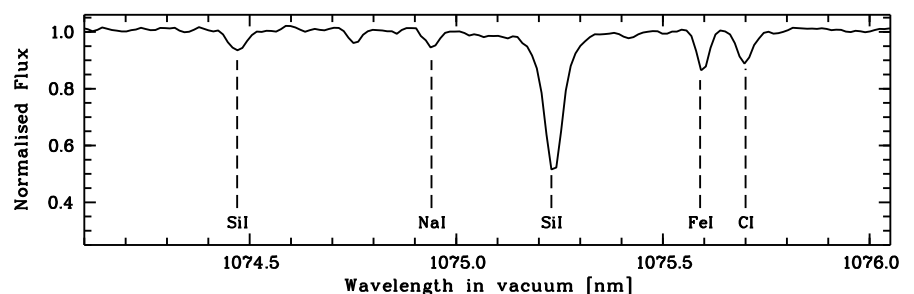

Fig. 8. The $\mathrm{Na}$ I line at $1075 \mathrm{~nm}$ (solid black) in the case of the star HD 24040.

We used three lines of Na I to derive the Na abundances; according to our calculations, they all form close to LTE and therefore we do not expect large NLTE corrections (see in Fig. 8 the line at $1075 \mathrm{~nm}$ for HD 24040). The NLTE atomic model of sodium was presented by Korotin \& Mishenina (1999) and then updated by Dobrovolskas et al. (2014). The updated sodium model currently consists of twenty energy levels of $\mathrm{Na}$ I and the ground level of $\mathrm{Na}$ II. In total, 46 radiative transitions were taken into account for the calculation of the population of all levels. We carried out test calculations for a grid of stellar atmosphere models in the ranges: $5000<T_{\text {eff }}<6000 \mathrm{~K}$ and $3.0<\log g<4.5$ with solar metallicity. For the IR sodium lines, NLTE effects lead to a slight increase in EW by $1-3 \%$, while the EW in the optical lines increases by $10-18 \%$. For all but one star we were able to derive the $\mathrm{Na}$ abundance with $\langle[\mathrm{Na} / \mathrm{Fe}]\rangle=+0.05 \pm 0.17$ in the sample. The large scatter is due to six stars with $[\mathrm{Na} / \mathrm{Fe}]$ around +0.1 dex and several stars with negative $[\mathrm{Na} / \mathrm{Fe}]$, down to about -0.2 dex.

The $\mathrm{Mg}$ I lines we selected to derive the $\mathrm{Mg}$ abundance are formed close to the LTE condition. In the case of $\mathrm{Mg}$, we used the model atom from Mishenina et al. (2004), then updated by Černiauskas et al. (2017). It consisted of 84 levels of Mg I, 12 levels of $\mathrm{Mg}$ II, and the ground state of Mg III. In the computation of departure coefficients, the radiative transitions between the first 59 levels of $\mathrm{Mg}$ I and the ground level of $\mathrm{Mg}$ II were taken into account. We carried out test calculations within the same grid of star parameters as for sodium. It turned out that due to the influence of the effects of the deviation from the LTE, the EW of the magnesium lines used in this analysis vary by $\pm 1 \%$; that is, these lines are formed almost in LTE. The average $[\mathrm{Mg} / \mathrm{Fe}]$ we derived $(\langle[\mathrm{Mg} / \mathrm{Fe}]\rangle=+0.02 \pm 0.07)$ is the one expected for disc stars around solar metallicity.

For Al, we investigated two lines at 1087.59 and $1676.79 \mathrm{~nm}$, which are formed close to LTE. Our Al atomic model is described in detail in Andrievsky et al. (2008). This model atom consists of 76 levels of $\mathrm{Al} \mathrm{I}$ and 13 levels of Al II. As shown by test calculations within that same grid star parameters as for the other NLTE calculations, Al I lines 1087 and $1676 \mathrm{~nm}$ respond very poorly to NLTE effects. The effect on EW is within $\pm 4 \%$, depending on the parameters of the atmosphere. For all stars but three, $A(\mathrm{Al})$ is based only on the $\mathrm{Al}$ I line at $1087.59 \mathrm{~nm}$; this line is weak but is in a range that is not overly contaminated by telluric absorption (see Fig. 9). For the three stars for which also the line at $1676.79 \mathrm{~nm}$ is kept, the agreement in $A(\mathrm{Al})$ derived from the two lines is good. For the 40 stars, we find $\langle[\mathrm{Al} / \mathrm{Fe}]\rangle=+0.10 \pm 0.06$, with some stars with $[\mathrm{Al} / \mathrm{Fe}]$ of about +0.2 dex. Only one star, HD 136618 , has a negative $[\mathrm{Al} / \mathrm{Fe}]$; this remains compatible within uncertainties with a solar ratio.

Five lines of K I have been analysed, at 1177.28, 1177.50, $1243.56,1252.55$, and $1516.72 \mathrm{~nm}$. The model atom of $\mathrm{K}$ was taken from Andrievsky et al. (2010) and consisted of 20 levels of K I and the ground level of K II. In addition, another 15 levels

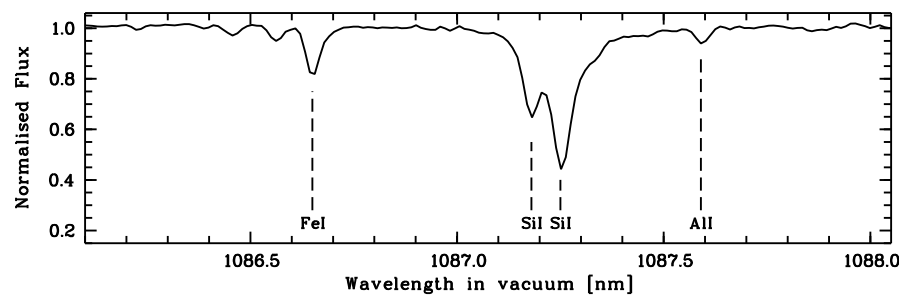

Fig. 9. The $\mathrm{Al} \mathrm{I}$ line at $1087 \mathrm{~nm}$ (solid black) in the case of the star HD 24040.

of $\mathrm{KI}$ and 7 levels of K II were used to ensure particle number conservation. The total number of bound-bound radiative transitions taken into account was 62 (see Andrievsky et al. 2010, for further details). Infrared lines of potassium showed a strong susceptibility to the influence of NLTE effects. Therefore, due to deviations from the LTE, the EW of the lines used increased from 10 to $32 \%$. We were able to derive $A(\mathrm{~K})$ for all but two stars, and $\langle[\mathrm{K} / \mathrm{Fe}]\rangle=+0.16 \pm 0.06$ for the 38 stars for which $\mathrm{K}$ has been derived. All the lines are sensitive to NLTE, and therefore the above-solar [K/Fe] value can easily be attributed to our LTE assumption.

$A(\mathrm{Ca})$ from $\mathrm{Ca} \mathrm{I}$ and $\mathrm{Ca}$ II lines is in good agreement $(\langle A(\mathrm{Ca} \mathrm{I})-A(\mathrm{Ca} I \mathrm{I})\rangle=-0.04 \pm 0.08)$ with a slightly higher value of $A(\mathrm{Ca})$ when derived from the $\mathrm{Ca}$ II lines, which can be explaind by the fact that the $\mathrm{Ca}$ I lines we selected to derive the $\mathrm{Ca}$ abundance are close to LTE while some of the Ca II lines are sensitive to NLTE and the $A(\mathrm{Ca})$ derived in LTE overestimates the abundance. However, the $A(\mathrm{Ca})$ abundance we derived for the sample stars is slightly high: from $\mathrm{Ca}$ I lines, $\langle[\mathrm{Ca} / \mathrm{Fe}]\rangle=$ $+0.12 \pm 0.05$. In particular for four stars, HD 97645, HD 99492, HD 147231 and HD 190360, [Ca/Fe] $>0.2$. The latter star of the four is overabundant in several elements (see below).

The $\mathrm{Ti}$ abundances from $\mathrm{Ti} \mathrm{I}$ and $\mathrm{Ti} I \mathrm{II}$ lines $(\langle A(\mathrm{Ti} \mathrm{I})-A(\mathrm{Ti} \mathrm{II})\rangle=+0.10 \pm 0.08)$ are well in agreement within uncertainties, and the $[\mathrm{Ti} / \mathrm{Fe}]$ for the stars analysed from Ti I lines is $\langle[\mathrm{Ti} / \mathrm{Fe}]\rangle=+0.09 \pm 0.05$. HD 116321 is rich in Ti.

All three Sr II lines already investigated in Caffau et al. (2016) are affected by NLTE. The high $[\mathrm{Sr} / \mathrm{Fe}]$ we derive for the 37 stars for which we derive $A(\mathrm{Sr})(\langle[\mathrm{Sr} / \mathrm{Fe}]\rangle=+0.42 \pm 0.12)$ is due to NLTE effects.

One star, HD 190360, has [P/Fe], [C/Fe], [S/Fe], and [Ca/Fe] ratios that are clearly high with respect to the solar pattern. This could be due to an underestimated effective temperature, HD 190360 being the only star for which the temperature is derived from a single calibration. Increasing the temperature by $180 \mathrm{~K}$ to match for example the $T_{\text {eff }}$ of Mishenina et al. (2004) of $5606 \mathrm{~K}$, the star no longer shows overabundance in $\mathrm{C}$, $\mathrm{P}$, and $\mathrm{S}$, but still shows an overabundance in $\mathrm{Ca}$. Also, Bensby et al. (2003) with an effective temperature of $5490 \mathrm{~K}$ and a surface gravity of 4.23 (values very similar to the ones we adopted) found an overabundance with respect to Fe of several elements. In Bensby et al. (2014), however, with a $T_{\text {eff }}=5572 \mathrm{~K}$, this overabundance is no longer present. It is interesting to note that a linear regression of the mean $T_{\text {eff }}$ over the $B-V T_{\text {eff }}$ for the 37 stars for which five temperature determinations are available predicts $T_{\text {eff }}=5593 \mathrm{~K}$ very close to the values of Mishenina et al. (2004) and Bensby et al. (2014).

\subsection{Helium}

The triplet line of neutral He arising from the high-excitation $(19.72 \mathrm{eV})$ metastable level $2 \mathrm{~s}^{3} \mathrm{~S}_{1}$ is observed in absorption in 
Table 3. Equivalent widths of the He I $1083 \mathrm{~nm}$ line.

\begin{tabular}{|c|c|c|c|}
\hline Star & $\mathrm{EW}(\mathrm{pm})$ & $\log _{10}\left(f_{X} / f_{\mathrm{Bol}}\right)$ & $d^{a}\left({ }^{\prime \prime}\right)$ \\
\hline HD 20670 & $\sim 2$ & & \\
\hline HD 24040 & $<5$ & & \\
\hline HD 28005 & $<10$ & & \\
\hline HD 32673 & $<10$ & & \\
\hline HD 34445 & 2 & & \\
\hline HD 34575 & 2 & & \\
\hline HD 44420 & $<\overline{5}$ & & \\
\hline HD 56303 & $<10$ & & \\
\hline HD 67346 & $<5$ & & \\
\hline HD 69056 & $<10$ & & \\
\hline HD 69809 & $<10$ & & \\
\hline HD 69960 & $<10$ & & \\
\hline HD 73226 & $<10$ & & \\
\hline HD 73933 & 11 & & \\
\hline HD 76909 & $<5$ & & \\
\hline HD 77519 & $<5$ & & \\
\hline HD 82943 & $<10$ & & \\
\hline HD 85301 & 20 & -3.29 & 15.31 \\
\hline HD 87359 & 6 & & \\
\hline HD 87836 & 3 & & \\
\hline HD 90681 & 8 & & \\
\hline HD 90722 & $<10$ & & \\
\hline HD 92788 & 4 & & \\
\hline HD 97645 & $<5$ & & \\
\hline HD 98618 & $<10$ & & \\
\hline HD 98736 & 7 & & \\
\hline HD 99491 & 7 & -4.12 & 26.17 \\
\hline HD 99492 & 11 & -3.72 & 50.60 \\
\hline HD 100069 & $<5$ & & \\
\hline HD 105631 & 15 & -3.45 & 1.96 \\
\hline HD 106116 & $<5$ & & \\
\hline HD 106156 & 12 & & \\
\hline HD 108942 & 3 & & \\
\hline HD 114174 & $<5$ & & \\
\hline HD 116321 & $<5$ & & \\
\hline HD 136618 & $<5$ & & \\
\hline HD 145675 & 6 & & \\
\hline HD 147231 & $<5$ & & \\
\hline HD 159222 & 8 & -4.06 & 18.85 \\
\hline HD 161797 & $<5$ & & \\
\hline
\end{tabular}

Notes. ${ }^{(a)}$ Angular distance between the X-ray source and the star.

the Sun and in solar-type stars. It is of too high an excitation to be formed in the photosphere and therefore must be formed in the chromosphere and very likely excited by radiation from the corona. In spatially resolved spectra of the Sun it can be observed in absorption in active regions (plages). Following the activity cycle of the Sun, the EW measured in the flux-integrated spectrum varies depending on the number of active regions. Andretta \& Giampapa (1995) suggested to use this line together with He I $587.6 \mathrm{~nm}$ to estimate the fraction of the stellar surface covered by active regions. In a pioneering work, Zirin (1982) obtained IR spectra at the coude focus of the Palomar five-metre telescope using image cathode tubes and photographic plates and films as detectors for 455 stars. The EW of the He I $1083.0 \mathrm{~nm}$ line is well correlated with the soft X-ray luminosity and in spite of the technical advances even today, the "observational data on the $\lambda 10830$ triplet line remains sparse" (verbatim from Smith 2016). This line has not been studied in great detail because it

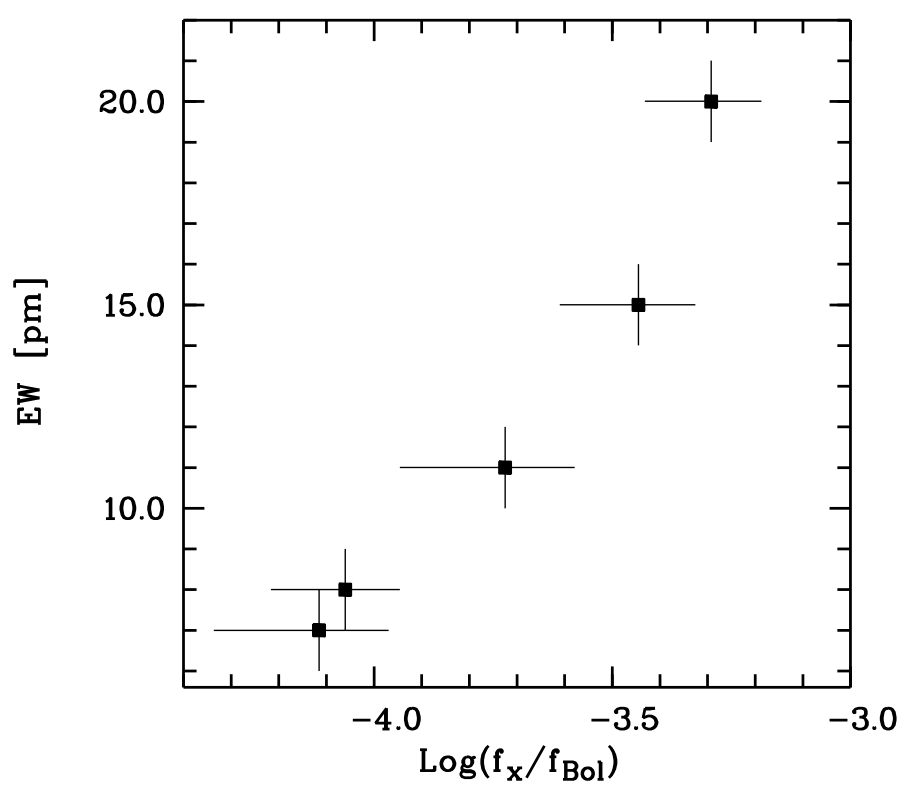

Fig. 10. The EW of the He I line vs. the logarithm of the ratio of $X$ and bolometric flux.

requires high-resolution observations. The large spectral coverage provided by GIANO ensures that the spectral range is always observed, and it lies in a region that is contaminated by a few telluric lines that sometimes affect the He line. We inspected all the spectra of our sample and were able to detect this line for 16 stars. When the line was detected we measured its EW (Table 3). The typical uncertainty is $1 \mathrm{pm}$. For five stars in our sample we found $\mathrm{X}$-ray measurements in the All-Sky Survey Faint source Catalogue (Voges et al. 2000). From these we derived $\log \left(f_{x} / f_{\text {bol }}\right)$, also provided in Table 3. The apparent bolometric magnitude was derived from the Gaia $G$ magnitude and the bolometric corrections of Bonifacio et al. (2018). The bolometric flux was then derived as recommended in IAU resolution 2015 B2 ${ }^{4}$ Eq. (4). These values are plotted in Fig. 10 against the EW of the He I $1083.0 \mathrm{~nm}$ line and a very good correlation can be seen between the two quantities. GIANO observations offer a good opportunity to monitor the activity cycles of solar-type stars from the observation of this line. This line may also be used to monitor the activity of stars with candidate planets found in radial-velocity searches. The monitoring of the He I $1083.0 \mathrm{~nm}$ line should allow us to derive the activity cycle of the star. This may help decipher whether radial velocity variations are due to planets or stellar activity. A well known example is HD 166435 (Queloz et al. 2001).

\section{Conclusions}

In this work, we analysed 40 stars for which we provided detailed chemical abundances of 14 elements. These stars belong to the Galactic disc and the abundances we derive are consistent with the chemical behaviour expected for Galactic disc stars; they are also in line with previous investigations (e.g. see Mikolaitis et al. 2014; Smiljanic et al. 2014, 2016; Kordopatis et al. 2015; Duffau et al. 2017). For some of the stars investigated here there are no detailed chemical analyses in the literature.

We were also able to add 40 values for the $\mathrm{P}$ abundance in Galactic stars, which is still a rather poorly studied element. We

\footnotetext{
4 https://WwW.iau.org/static/resolutions/IAU2015_ English.pdf
} 
also introduced a PI line of Mult. 4, the abundance of which is consistent with, though slightly higher than, the $\mathrm{P}$ abundance derived from the PI lines of Mult. 1, used in previous investigations (Caffau et al. 2011, 2016).

For a subsample of nine stars, we compared the abundances derived from the IR GIANO spectra and SOPHIE optical spectra. For eight chemical abundances common to both surveys agreement was found to be very good. Nevertheless, the line-toline scatter in the IR spectra is larger than in the optical spectra, probably due to a better knowledge on atomic data in the latter range and this is probably related to the longer history of use of optical ranges.

Both the good agreement on the abundances derived from IR and optical spectra and the good agreement with the literature are indicators that the abundances we derive from GIANO spectra are reliable. For the star HD 98736, we analysed only four orders of GIANO (47-50) which correspond to the highresolution mode of the $H$-band for MOONS. In this case we can derive abundances for seven elements $(\mathrm{C}, \mathrm{Si}, \mathrm{S}, \mathrm{Ti}, \mathrm{Cr}, \mathrm{Fe}$ and $\mathrm{Ni}$ ). We analysed this range also for the star HD 24040, the spectral range shown in Fig. 1, and we derive the abundance for $\mathrm{C}$, $\mathrm{Si}, \mathrm{S}, \mathrm{K}, \mathrm{Ti}, \mathrm{Cr}, \mathrm{Fe}$, Ni. Clearly, by using only four orders of the GIANO spectra, the uncertainties in the abundances are much larger because the number of lines available is much lower than when analysing the complete GIANO range; for the majority of the elements abundances are based on a single line. On top of that, because of the lower resolution that MOONS will have with respect to GIANO, some of these elements will be difficult to be derived by MOONS, in particular $\mathrm{Ti}, \mathrm{Cr}$, and $\mathrm{Ni}$.

For several stars in the sample we were able to detect the He I line at $1083.0 \mathrm{~nm}$ and confirm that its EW is strongly correlated with the logarithmic ratio of X-ray flux to bolometric flux and, hence, stellar activity. Since He measurements in dwarf stars are still limited in number, GIANO provides a great opportunity in regards to this topic.

GIANO provides us with a great chance to investigate Galactic stars and to derive their detailed chemical abundances. From GIANO spectra we could derive the abundances of some elements (C, P, K, Sr) that could not be derived from the SOPHIE optical spectra. GIANO's high resolution and large spectral range are fundamental to deriving trustable abundances for several elements. GIANO is a single-object spectrograph meaning that stars can only be observed one at a time, but in the next years its archive will fill and the number of stars available will be larger and larger.

Acknowledgements. We are grateful to the referee, Mathieu Van der Swaelmen, for his suggestions and comments which made the paper more readable and understandable. E.C. and P.B. have been supported by the Programme National de Physique Stellaire of the Institut National des Sciences de l'Univers of CNRS. S.D. acknowledges support from Comité Mixto ESO-Gobierno de Chile.

\section{References}

Allende Prieto, C., Majewski, S. R., Schiavon, R., et al. 2008, Astron. Nachr., 329, 1018

Andretta, V., \& Giampapa, M. S. 1995, ApJ, 439, 405

Andrievsky, S. M., Spite, M., Korotin, S. A., et al. 2008, A\&A, 481, 481

Andrievsky, S. M., Spite, M., Korotin, S. A., et al. 2010, A\&A, 509, A88

Behara, N. T., Bonifacio, P., Ludwig, H.-G., et al. 2010, A\&A, 513, A72

Bensby, T., Feltzing, S., \& Lundström, I. 2003, A\&A, 410, 527
Bensby, T., Feltzing, S., \& Oey, M. S. 2014, A\&A, 562, A71

Boeche, C., \& Grebel, E. K. 2016, A\&A, 587, A2

Bonifacio, P., Caffau, E., Ludwig, H.-G., et al. 2018, A\&A, 611, A68

Bouchy, F., \& Sophie Team 2006, Tenth Anniversary of 51 Peg-b: Status of and Prospects for Hot Jupiter Studies, 319

Brewer, J. M., Fischer, D. A., Valenti, J. A., \& Piskunov, N. 2016, ApJS, 225, 32 Caffau, E., \& Ludwig, H.-G. 2007, A\&A, 467, L11

Caffau, E., Bonifacio, P., Faraggiana, R., \& Steffen, M. 2011, A\&A, 532, A98

Caffau, E., Andrievsky, S., Korotin, S., et al. 2016, A\&A, 585, A16

Capitanio, L., Lallement, R., Vergely, J. L., Elyajouri, M., \& Monreal-Ibero, A. 2017, A\&A, 606, A65

Casagrande, L., Schönrich, R., Asplund, M., et al. 2011, A\&A, 530, A138

Černiauskas, A., Kučinskas, A., Klevas, J., et al. 2017, A\&A, 604, A35

Chen, Y. Q., Zhao, G., Izumiura, H., et al. 2008, AJ, 135, 618

Cirasuolo, M., Afonso, J., Carollo, M., et al. 2014, Proc. SPIE, 9147, 91470N

Cutri, R. M., Skrutskie, M. F., Van Dyk, S., et al. 2003, The IRSA 2MASS All-Sky Point Source Catalog, NASA/IPAC Infrared Science Archive, http://www.ipac.caltech.edu/2mass/releases/allsky/ doc/explsup.html

da Silva, R., Milone, A. d. C., \& Rocha-Pinto, H. J. 2015, A\&A, 580, A24

Dalton, G., Trager, S., Abrams, D. C., et al. 2016, Proc. SPIE, 9908, $99081 \mathrm{G}$

Dekker, H., D’Odorico, S., Kaufer, A., Delabre, B., \& Kotzlowski, H. 2000, Proc. SPIE, 4008, 534

de Jong, R. S., Barden, S., Bellido-Tirado, O., et al. 2014, Proc. SPIE, 9147, 91470M

Dobrovolskas, V., Kučinskas, A., Bonifacio, P., et al. 2014, A\&A, 565, A121

Duffau, S., Caffau, E., Sbordone, L., et al. 2017, A\&A, 604, A128

Ecuvillon, A., Israelian, G., Santos, N. C., et al. 2004, A\&A, 426, 619

ESA 1997, The HIPPARCOS and Tycho catalogues. Astrometric and photometric star catalogues derived from the ESA HIPPARCOS Space Astrometry Mission (Noordwijk: Netherlands), ESA SP, 1200

Gaia Collaboration (Babusiaux, C., et al.) 2018a, A\&A, 616, A10

Gaia Collaboration (Brown, A. G. A., et al.) 2018b, A\&A, 616, A1

Gilmore, G., Randich, S., Asplund, M., et al. 2012, The Messenger, 147, 25

González Hernández, J. I., \& Bonifacio, P. 2009, A\&A, 497, 497

Hayek, W., Asplund, M., Collet, R., \& Nordlund, Å. 2011, A\&A, 529, A158

Kaeufl, H.-U., Ballester, P., Biereichel, P., et al. 2004, Proc. SPIE, 5492, 1218

Kordopatis, G., Wyse, R. F. G., Gilmore, G., et al. 2015, A\&A, 582, A122

Korotin, S. A., \& Mishenina, T. V. 1999, Astron. Rep., 43, 533

Kurucz, R. L. 2005, Mem. Soc. Astron. It. Suppl., 8, 14

Ludwig, H.-G., Caffau, E., Steffen, M., et al. 2009, Mem. Soc. Astron. It., 80, 711

Magic, Z., Collet, R., Asplund, M., et al. 2013, A\&A, 557, A26

Masana, E., Jordi, C., \& Ribas, I. 2006, A\&A, 450, 735

Mayor, M., Pepe, F., Queloz, D., et al. 2003, The Messenger, 114, 20

Mikolaitis, Š., Hill, V., Recio-Blanco, A., et al. 2014, A\&A, 572, A33

Mishenina, T. V., Soubiran, C., Kovtyukh, V. V., \& Korotin, S. A. 2004, A\&A, 418, 551

Mishenina, T. V., Pignatari, M., Korotin, S. A., et al. 2013, A\&A, 552, A128

Molinaro, M., Calabria, N. F., Butora, R., Zorba, S., \& Smareglia, R. 2018, Proc. SPIE, 10707, 1070722

Oliva, E., Sanna, N., Rainer, M., et al. 2018, Proc. SPIE, 10702, 1070274

Origlia, L., Oliva, E., Baffa, C., et al. 2014, Proc. SPIE, 91446, 914461E

Pasquini, L., Avila, G., Blecha, A., et al. 2002, The Messenger, 110, 1

Petigura, E. A., \& Marcy, G. W. 2011, ApJ, 735, 41

Queloz, D., Henry, G. W., Sivan, J. P., et al. 2001, A\&A, 379, 279

Ramírez, I., Fish, J. R., Lambert, D. L., \& Allende Prieto C. 2012, ApJ, 756, 46

Roederer, I. U., Jacobson, H. R., Thanathibodee, T., Frebel, A., \& Toller, E. 2014, ApJ, 797, 69

Sbordone, L., Bonifacio, P., Castelli, F., \& Kurucz, R. L. 2004, Mem. Soc. Astron. It. Suppl., 5, 93

Sbordone, L., Caffau, E., Bonifacio, P., \& Duffau, S. 2014, A\&A, 564, A109

Shetrone, M., Bizyaev, D., Lawler, J. E., et al. 2015, ApJS, 221, 24

Smiljanic, R., Korn, A. J., Bergemann, M., et al. 2014, A\&A, 570, A122

Smiljanic, R., Romano, D., Bragaglia, A., et al. 2016, A\&A, 589, A115

Smith, G. H. 2016, PASA, 33, e057

Steffen, M., Caffau, E., \& Ludwig, H.-G. 2013, Mem. Soc. Astron. It. Suppl., 24, 37

Tozzi, A., Oliva, E., Origlia, L., et al. 2014, Proc. SPIE, 9147, 91479N

Tozzi, A., Oliva, E., Iuzzolino, M., et al. 2016, Proc. SPIE, 9908, 99086C

Valenti, J. A., \& Fischer, D. A. 2005, ApJS, 159, 141

Voges, W., Aschenbach, B., Boller, T., et al. 2000, IAU Circ., 7432, 1

Zirin, H. 1982, ApJ, 260, 655 
Appendix A: Stellar parameters and chemical abundances

Table A.1. Stellar parameters of our complete sample of stars.

\begin{tabular}{|c|c|c|c|c|c|c|c|}
\hline Star & $\begin{array}{l}T_{\text {eff }} \\
\mathrm{K}\end{array}$ & $\begin{array}{l}\log g \\
(\mathrm{cgs})\end{array}$ & {$[\mathrm{Fe} / \mathrm{H}]$} & $A(\mathrm{FeI})$ & $N$ lines & $A(\mathrm{FeII})$ & $N$ lines \\
\hline HD 20670 & $5688 \pm 138$ & 3.65 & 0.10 & $7.62 \pm 0.16$ & 76 & $7.63 \pm 0.09$ & 4 \\
\hline HD 24040 & $5809 \pm 136$ & 4.12 & 0.09 & $7.61 \pm 0.17$ & 81 & $7.60 \pm 0.07$ & 4 \\
\hline HD 28005 & $5802 \pm 144$ & 4.18 & 0.21 & $7.73 \pm 0.16$ & 71 & $7.73 \pm 0.09$ & 4 \\
\hline HD 32673 & $5752 \pm 135$ & 3.53 & 0.07 & $7.59 \pm 0.15$ & 73 & $7.53 \pm 0.09$ & 4 \\
\hline HD 34445 & $5803 \pm 112$ & 4.06 & -0.03 & $7.49 \pm 0.15$ & 76 & $7.51 \pm 0.11$ & 3 \\
\hline HD 34575 & $5582 \pm 134$ & 4.22 & 0.18 & $7.70 \pm 0.17$ & 60 & $7.74 \pm 0.06$ & 3 \\
\hline HD 44420 & $5777 \pm 151$ & 4.23 & 0.19 & $7.71 \pm 0.16$ & 65 & $7.68 \pm 0.09$ & 4 \\
\hline HD 56303 & $5941 \pm 125$ & 4.21 & 0.05 & $7.57 \pm 0.15$ & 90 & $7.59 \pm 0.09$ & 4 \\
\hline HD 67346 & $5953 \pm 140$ & 3.78 & 0.14 & $7.66 \pm 0.16$ & 80 & $7.66 \pm 0.11$ & 4 \\
\hline HD 69056 & $5637 \pm 138$ & 4.28 & 0.05 & $7.57 \pm 0.16$ & 78 & $7.55 \pm 0.12$ & 3 \\
\hline HD 69809 & $5842 \pm 147$ & 4.16 & 0.17 & $7.69 \pm 0.14$ & 75 & $7.68 \pm 0.14$ & 4 \\
\hline HD 69960 & $5655 \pm 147$ & 3.99 & 0.22 & $7.74 \pm 0.17$ & 62 & $7.67 \pm 0.06$ & 3 \\
\hline HD 73226 & $5886 \pm 148$ & 4.21 & 0.06 & $7.58 \pm 0.16$ & 81 & $7.54 \pm 0.14$ & 3 \\
\hline HD 73933 & $6143 \pm 134$ & 4.24 & 0.06 & $7.58 \pm 0.17$ & 83 & $7.49 \pm 0.14$ & 3 \\
\hline HD 76909 & $5655 \pm 159$ & 4.17 & 0.24 & $7.76 \pm 0.17$ & 60 & $7.82 \pm 0.06$ & 2 \\
\hline HD 77519 & $6140 \pm 190$ & 3.85 & 0.14 & $7.66 \pm 0.17$ & 94 & $7.66 \pm 0.12$ & 4 \\
\hline HD 82943 & $5917 \pm 284$ & 4.23 & 0.13 & $7.65 \pm 0.17$ & 84 & $7.70 \pm 0.05$ & 4 \\
\hline HD 85301 & $5640 \pm 128$ & 4.44 & 0.05 & $7.57 \pm 0.19$ & 70 & $7.61 \pm 0.02$ & 2 \\
\hline HD 87359 & $5645 \pm 140$ & 4.40 & -0.07 & $7.45 \pm 0.17$ & 78 & $7.50 \pm 0.05$ & 4 \\
\hline HD 87836 & $5684 \pm 158$ & 4.05 & 0.16 & $7.68 \pm 0.15$ & 58 & $7.63 \pm 0.17$ & 4 \\
\hline HD 90681 & $5950 \pm 167$ & 4.26 & 0.16 & $7.68 \pm 0.16$ & 78 & $7.68 \pm 0.09$ & 4 \\
\hline HD 90722 & $5677 \pm 141$ & 4.12 & 0.22 & $7.74 \pm 0.16$ & 57 & 7.70 & 1 \\
\hline HD 92788 & $5694 \pm 196$ & 4.26 & 0.13 & $7.65 \pm 0.17$ & 68 & $7.67 \pm 0.16$ & 4 \\
\hline HD 97645 & $6127 \pm 125$ & 4.10 & 0.14 & $7.66 \pm 0.18$ & 81 & $7.62 \pm 0.09$ & 4 \\
\hline HD 98618 & $5727 \pm 138$ & 4.27 & -0.11 & $7.41 \pm 0.17$ & 89 & $7.45 \pm 0.05$ & 3 \\
\hline HD 98736 & $5276 \pm 100$ & 4.40 & 0.29 & $7.81 \pm 0.19$ & 49 & $7.90 \pm 0.13$ & 3 \\
\hline HD 99491 & $5537 \pm 178$ & 4.40 & 0.25 & $7.77 \pm 0.18$ & 58 & $7.76 \pm 0.14$ & 2 \\
\hline HD 99492 & $5006 \pm 235$ & 4.56 & 0.20 & $7.72 \pm 0.23$ & 52 & $7.75 \pm 0.12$ & 2 \\
\hline HD 100069 & $5796 \pm 129$ & 3.74 & -0.03 & $7.49 \pm 0.18$ & 79 & $7.45 \pm 0.15$ & 3 \\
\hline HD 105631 & $5391 \pm 104$ & 4.47 & 0.05 & $7.57 \pm 0.18$ & 62 & $7.61 \pm 0.05$ & 2 \\
\hline HD 106116 & $5665 \pm 164$ & 4.30 & 0.03 & $7.55 \pm 0.16$ & 70 & $7.61 \pm 0.11$ & 3 \\
\hline HD 106156 & $5449 \pm 175$ & 4.45 & 0.10 & $7.62 \pm 0.17$ & 61 & 7.67 & 1 \\
\hline HD 108942 & $5882 \pm 179$ & 4.27 & 0.20 & $7.72 \pm 0.18$ & 74 & $7.66 \pm 0.10$ & 4 \\
\hline HD 114174 & $5728 \pm 193$ & 4.30 & -0.06 & $7.46 \pm 0.17$ & 74 & $7.48 \pm 0.08$ & 3 \\
\hline HD 116321 & $6292 \pm 188$ & 3.66 & 0.10 & $7.62 \pm 0.20$ & 81 & $7.56 \pm 0.10$ & 3 \\
\hline HD 136618 & $5805 \pm 125$ & 3.56 & 0.14 & $7.66 \pm 0.18$ & 73 & $7.62 \pm 0.15$ & 4 \\
\hline HD 145675 & $5312 \pm 149$ & 4.37 & 0.33 & $7.85 \pm 0.18$ & 46 & $7.92 \pm 0.05$ & 2 \\
\hline HD 147231 & $5594 \pm 132$ & 4.30 & -0.14 & $7.38 \pm 0.17$ & 78 & $7.48 \pm 0.09$ & 3 \\
\hline HD 159222 & $5815 \pm 125$ & 4.30 & 0.00 & $7.52 \pm 0.16$ & 76 & $7.57 \pm 0.11$ & 4 \\
\hline HD 190360 & $5424 \pm 250$ & 4.21 & -0.01 & $7.51 \pm 0.16$ & 57 & $7.59 \pm 0.06$ & 3 \\
\hline
\end{tabular}

Notes. The uncertainty in gravity is smaller than $0.1 \mathrm{dex}$ for all stars. 
E. Caffau et al.: Systematic investigation of chemical abundances derived using IR spectra obtained with GIANO

Table A.2. Abundances for $\mathrm{C}, \mathrm{Na}, \mathrm{Mg}$, and $\mathrm{Al}$ for our complete sample of stars.

\begin{tabular}{|c|c|c|c|c|c|c|c|c|}
\hline$\underline{\text { Star }}$ & $A(\mathrm{C})$ & $N$ lines & $A(\mathrm{Na})$ & $N$ lines & $A(\mathrm{Mg})$ & $N$ lines & $A(\mathrm{Al})$ & $N$ lines \\
\hline HD 20670 & $8.50 \pm 0.22$ & 22 & $6.41 \pm 0.07$ & 3 & $7.69 \pm 0.11$ & 10 & 6.71 & 1 \\
\hline HD 24040 & $8.48 \pm 0.21$ & 22 & $6.33 \pm 0.07$ & 2 & $7.68 \pm 0.13$ & 10 & 6.62 & 1 \\
\hline HD 28005 & $8.68 \pm 0.23$ & 19 & 6.60 & 1 & $7.81 \pm 0.13$ & 8 & 6.85 & 1 \\
\hline HD 32673 & $8.44 \pm 0.23$ & 22 & $6.35 \pm 0.06$ & 2 & $7.69 \pm 0.08$ & 8 & 6.57 & 1 \\
\hline HD 34445 & $8.44 \pm 0.22$ & 24 & 6.22 & 1 & $7.56 \pm 0.12$ & 8 & 6.49 & 1 \\
\hline HD 34575 & $8.64 \pm 0.24$ & 23 & $6.42 \pm 0.11$ & 2 & $7.82 \pm 0.09$ & 8 & 6.78 & 1 \\
\hline HD 44420 & $8.65 \pm 0.24$ & 21 & $6.42 \pm 0.19$ & 2 & $7.80 \pm 0.10$ & 9 & 6.87 & 1 \\
\hline HD 56303 & $8.44 \pm 0.19$ & 25 & $6.19 \pm 0.03$ & 2 & $7.65 \pm 0.10$ & 9 & 6.56 & 1 \\
\hline HD 67346 & $8.61 \pm 0.20$ & 21 & $6.52 \pm 0.09$ & 2 & $7.70 \pm 0.14$ & 10 & 6.63 & 1 \\
\hline HD 69056 & $8.50 \pm 0.22$ & 25 & $6.22 \pm 0.15$ & 3 & $7.69 \pm 0.12$ & 10 & 6.69 & 1 \\
\hline HD 69809 & $8.65 \pm 0.22$ & 19 & 6.44 & 1 & $7.73 \pm 0.13$ & 11 & 6.85 & 1 \\
\hline HD 69960 & $8.64 \pm 0.22$ & 21 & $6.29 \pm 0.15$ & 2 & $7.66 \pm 0.29$ & 8 & 6.83 & 1 \\
\hline HD 73226 & $8.48 \pm 0.21$ & 24 & $6.24 \pm 0.23$ & 3 & $7.60 \pm 0.14$ & 7 & 6.68 & 1 \\
\hline HD 73933 & $8.45 \pm 0.21$ & 24 & $6.17 \pm 0.00$ & 2 & $7.59 \pm 0.14$ & 12 & $6.59 \pm 0.11$ & 2 \\
\hline HD 76909 & $8.73 \pm 0.20$ & 19 & 6.51 & 1 & $7.82 \pm 0.12$ & 8 & 6.81 & 1 \\
\hline HD 77519 & $8.57 \pm 0.23$ & 20 & $6.30 \pm 0.19$ & 3 & $7.68 \pm 0.14$ & 10 & $6.68 \pm 0.12$ & 2 \\
\hline HD 82943 & $8.57 \pm 0.21$ & 21 & $6.40 \pm 0.07$ & 2 & $7.67 \pm 0.14$ & 9 & 6.72 & 1 \\
\hline HD 85301 & $8.45 \pm 0.21$ & 30 & $6.08 \pm 0.03$ & 2 & $7.53 \pm 0.18$ & 9 & 6.59 & 1 \\
\hline HD 87359 & $8.36 \pm 0.19$ & 34 & $6.13 \pm 0.06$ & 2 & $7.49 \pm 0.11$ & 10 & 6.40 & 1 \\
\hline HD 87836 & $8.66 \pm 0.26$ & 23 & 6.60 & 1 & $7.73 \pm 0.14$ & 8 & 6.77 & 1 \\
\hline HD 90681 & $8.55 \pm 0.21$ & 22 & $6.34 \pm 0.16$ & 3 & $7.73 \pm 0.14$ & 10 & 6.68 & 1 \\
\hline HD 90722 & $8.73 \pm 0.21$ & 21 & 6.50 & 1 & $7.80 \pm 0.09$ & 6 & 6.78 & 1 \\
\hline HD 92788 & $8.63 \pm 0.17$ & 21 & $6.46 \pm 0.15$ & 2 & $7.71 \pm 0.17$ & 11 & 6.79 & 1 \\
\hline HD 97645 & $8.48 \pm 0.22$ & 21 & $6.27 \pm 0.00$ & 2 & $7.66 \pm 0.11$ & 11 & $6.75 \pm 0.02$ & 2 \\
\hline HD 98618 & $8.41 \pm 0.21$ & 27 & $6.13 \pm 0.22$ & 3 & $7.49 \pm 0.11$ & 11 & 6.42 & 1 \\
\hline HD 98736 & $8.84 \pm 0.24$ & 27 & 6.72 & 1 & $7.81 \pm 0.15$ & 6 & 6.89 & 1 \\
\hline HD 99491 & $8.64 \pm 0.17$ & 26 & $6.53 \pm 0.18$ & 2 & $7.77 \pm 0.18$ & 8 & 6.86 & 1 \\
\hline HD 99492 & $8.71 \pm 0.35$ & 28 & $6.62 \pm 0.14$ & 2 & $7.68 \pm 0.21$ & 5 & 6.90 & 1 \\
\hline HD 100069 & $8.45 \pm 0.22$ & 22 & $6.19 \pm 0.08$ & 3 & $7.37 \pm 0.48$ & 7 & 6.58 & 1 \\
\hline HD 105631 & $8.49 \pm 0.23$ & 32 & $6.27 \pm 0.02$ & 2 & $7.57 \pm 0.15$ & 9 & 6.54 & 1 \\
\hline HD 106116 & $8.50 \pm 0.16$ & 25 & $6.27 \pm 0.11$ & 3 & $7.43 \pm 0.51$ & 7 & 6.66 & 1 \\
\hline HD 106156 & $8.60 \pm 0.16$ & 26 & $6.32 \pm 0.07$ & 3 & $7.59 \pm 0.15$ & 8 & 6.64 & 1 \\
\hline HD 108942 & $8.57 \pm 0.23$ & 22 & 6.36 & 1 & $7.73 \pm 0.14$ & 10 & 6.75 & 1 \\
\hline HD 114174 & $8.47 \pm 0.20$ & 25 & $6.23 \pm 0.12$ & 2 & $7.38 \pm 0.42$ & 7 & 6.50 & 1 \\
\hline HD 116321 & $8.55 \pm 0.24$ & 17 & & 0 & $7.67 \pm 0.12$ & 8 & 6.71 & 1 \\
\hline HD 136618 & $8.59 \pm 0.20$ & 18 & 6.45 & 1 & $7.76 \pm 0.14$ & 9 & 6.53 & 1 \\
\hline HD 145675 & $8.84 \pm 0.22$ & 27 & 6.76 & 1 & $7.90 \pm 0.11$ & 4 & 6.94 & 1 \\
\hline HD 147231 & $8.47 \pm 0.25$ & 29 & $6.06 \pm 0.17$ & 3 & $7.56 \pm 0.11$ & 9 & 6.40 & 1 \\
\hline HD 159222 & $8.47 \pm 0.22$ & 22 & $6.16 \pm 0.07$ & 3 & $7.54 \pm 0.18$ & 9 & 6.53 & 1 \\
\hline HD 190360 & $8.84 \pm 0.16$ & 21 & $6.26 \pm 0.08$ & 2 & $7.67 \pm 0.10$ & 4 & 6.64 & 1 \\
\hline
\end{tabular}


Table A.3. Same as Table A.2 for Si, P, S, and K.

\begin{tabular}{|c|c|c|c|c|c|c|c|c|}
\hline Star & $A(\mathrm{Si})$ & $N$ lines & $A(\mathrm{P})$ & $N$ lines & $A(\mathrm{~S})$ & $N$ lines & $A(\mathrm{~K})$ & $N$ lines \\
\hline HD 20670 & $7.61 \pm 0.24$ & 28 & $5.65 \pm 0.12$ & 2 & $7.27 \pm 0.15$ & 8 & $5.39 \pm 0.08$ & 3 \\
\hline HD 24040 & $7.61 \pm 0.23$ & 28 & $5.60 \pm 0.13$ & 3 & $7.25 \pm 0.17$ & 8 & $5.37 \pm 0.22$ & 5 \\
\hline HD 28005 & $7.70 \pm 0.23$ & 24 & $5.79 \pm 0.10$ & 3 & $7.35 \pm 0.17$ & 8 & $5.57 \pm 0.08$ & 2 \\
\hline HD 32673 & $7.56 \pm 0.22$ & 26 & $5.49 \pm 0.17$ & 3 & $7.28 \pm 0.21$ & 7 & $5.37 \pm 0.05$ & 3 \\
\hline HD 34445 & $7.54 \pm 0.20$ & 24 & $5.52 \pm 0.04$ & 3 & $7.24 \pm 0.12$ & 9 & $5.26 \pm 0.15$ & 4 \\
\hline HD 34575 & $7.65 \pm 0.23$ & 23 & $5.74 \pm 0.10$ & 3 & $7.42 \pm 0.17$ & 8 & $5.43 \pm 0.13$ & 3 \\
\hline HD 44420 & $7.71 \pm 0.23$ & 27 & $5.79 \pm 0.13$ & 3 & $7.47 \pm 0.18$ & 10 & $5.57 \pm 0.02$ & 2 \\
\hline HD 56303 & $7.56 \pm 0.23$ & 28 & $5.54 \pm 0.05$ & 3 & $7.21 \pm 0.13$ & 11 & $5.23 \pm 0.15$ & 4 \\
\hline HD 67346 & $7.70 \pm 0.25$ & 26 & $5.72 \pm 0.05$ & 3 & $7.46 \pm 0.18$ & 6 & $5.40 \pm 0.13$ & 4 \\
\hline HD 69056 & $7.58 \pm 0.23$ & 26 & $5.51 \pm 0.06$ & 3 & $7.16 \pm 0.17$ & 10 & $5.34 \pm 0.13$ & 4 \\
\hline HD 69809 & $7.70 \pm 0.24$ & 24 & $5.77 \pm 0.09$ & 3 & $7.34 \pm 0.08$ & 10 & $5.51 \pm 0.10$ & 3 \\
\hline HD 69960 & $7.72 \pm 0.27$ & 22 & $5.73 \pm 0.07$ & 3 & $7.30 \pm 0.18$ & 8 & $5.51 \pm 0.02$ & 2 \\
\hline HD 73226 & $7.63 \pm 0.22$ & 27 & $5.60 \pm 0.03$ & 3 & $7.28 \pm 0.17$ & 9 & $5.28 \pm 0.17$ & 3 \\
\hline HD 73933 & $7.63 \pm 0.21$ & 23 & $5.45 \pm 0.12$ & 2 & $7.21 \pm 0.16$ & 7 & $5.28 \pm 0.13$ & 4 \\
\hline HD 76909 & $7.74 \pm 0.26$ & 19 & $5.86 \pm 0.08$ & 3 & $7.39 \pm 0.20$ & 9 & $5.58 \pm 0.03$ & 2 \\
\hline HD 77519 & $7.68 \pm 0.26$ & 27 & $5.75 \pm 0.05$ & 3 & $7.37 \pm 0.15$ & 7 & $5.45 \pm 0.23$ & 5 \\
\hline HD 82943 & $7.67 \pm 0.24$ & 26 & $5.67 \pm 0.08$ & 3 & $7.33 \pm 0.18$ & 9 & $5.37 \pm 0.09$ & 4 \\
\hline HD 85301 & $7.59 \pm 0.25$ & 24 & $5.53 \pm 0.08$ & 2 & $7.29 \pm 0.24$ & 8 & $5.17 \pm 0.16$ & 3 \\
\hline HD 87359 & $7.50 \pm 0.23$ & 27 & $5.46 \pm 0.20$ & 3 & $7.12 \pm 0.16$ & 12 & $5.12 \pm 0.15$ & 4 \\
\hline HD 87836 & $7.70 \pm 0.25$ & 25 & $5.70 \pm 0.15$ & 3 & $7.32 \pm 0.17$ & 8 & $5.50 \pm 0.05$ & 2 \\
\hline HD 90681 & $7.70 \pm 0.25$ & 27 & $5.65 \pm 0.16$ & 3 & $7.35 \pm 0.17$ & 7 & $5.38 \pm 0.14$ & 4 \\
\hline HD 90722 & $7.69 \pm 0.26$ & 24 & $5.75 \pm 0.17$ & 3 & $7.41 \pm 0.12$ & 8 & $5.41 \pm 0.17$ & 2 \\
\hline HD 92788 & $7.69 \pm 0.25$ & 25 & $5.74 \pm 0.10$ & 3 & $7.36 \pm 0.17$ & 9 & $5.37 \pm 0.13$ & 3 \\
\hline HD 97645 & $7.72 \pm 0.23$ & 26 & $5.61 \pm 0.10$ & 3 & $7.28 \pm 0.19$ & 9 & $5.34 \pm 0.15$ & 4 \\
\hline HD 98618 & $7.49 \pm 0.21$ & 26 & $5.49 \pm 0.09$ & 3 & $7.16 \pm 0.22$ & 12 & $5.08 \pm 0.09$ & 4 \\
\hline HD 98736 & $7.85 \pm 0.25$ & 21 & $5.97 \pm 0.25$ & 3 & $7.52 \pm 0.18$ & 7 & 5.62 & 1 \\
\hline HD 99491 & $7.76 \pm 0.24$ & 21 & $5.65 \pm 0.24$ & 3 & $7.27 \pm 0.23$ & 9 & & 0 \\
\hline HD 99492 & $7.75 \pm 0.27$ & 24 & $5.72 \pm 0.24$ & 2 & $7.15 \pm 0.31$ & 6 & & 0 \\
\hline HD 100069 & $7.53 \pm 0.22$ & 27 & $5.53 \pm 0.09$ & 3 & $7.18 \pm 0.12$ & 9 & $5.30 \pm 0.23$ & 5 \\
\hline HD 105631 & $7.56 \pm 0.29$ & 24 & $5.64 \pm 0.36$ & 2 & $7.23 \pm 0.17$ & 11 & $5.22 \pm 0.19$ & 3 \\
\hline HD 106116 & $7.60 \pm 0.24$ & 24 & $5.64 \pm 0.06$ & 3 & $7.24 \pm 0.16$ & 10 & 5.30 & 1 \\
\hline HD 106156 & $7.64 \pm 0.25$ & 23 & $5.68 \pm 0.10$ & 2 & $7.33 \pm 0.16$ & 7 & $5.34 \pm 0.17$ & 3 \\
\hline HD 108942 & $7.68 \pm 0.26$ & 24 & $5.76 \pm 0.18$ & 3 & $7.24 \pm 0.16$ & 9 & $5.42 \pm 0.19$ & 3 \\
\hline HD 114174 & $7.52 \pm 0.24$ & 23 & $5.56 \pm 0.03$ & 3 & $7.11 \pm 0.19$ & 10 & $5.28 \pm 0.02$ & 3 \\
\hline HD 116321 & $7.69 \pm 0.22$ & 24 & $5.71 \pm 0.01$ & 2 & $7.37 \pm 0.21$ & 8 & $5.41 \pm 0.18$ & 5 \\
\hline HD 136618 & $7.67 \pm 0.26$ & 20 & $5.69 \pm 0.06$ & 2 & $7.24 \pm 0.22$ & 8 & $5.41 \pm 0.12$ & 3 \\
\hline HD 145675 & $7.91 \pm 0.30$ & 16 & $5.91 \pm 0.22$ & 3 & $7.49 \pm 0.17$ & 9 & 5.69 & 1 \\
\hline HD 147231 & $7.49 \pm 0.24$ & 22 & $5.52 \pm 0.09$ & 3 & $7.12 \pm 0.18$ & 10 & $5.23 \pm 0.15$ & 4 \\
\hline HD 159222 & $7.55 \pm 0.21$ & 22 & $5.53 \pm 0.05$ & 3 & $7.22 \pm 0.16$ & 8 & $5.37 \pm 0.10$ & 4 \\
\hline HD 190360 & $7.67 \pm 0.29$ & 20 & $6.01 \pm 0.38$ & 2 & $7.52 \pm 0.11$ & 4 & $5.29 \pm 0.15$ & 3 \\
\hline
\end{tabular}


E. Caffau et al.: Systematic investigation of chemical abundances derived using IR spectra obtained with GIANO

Table A.4. Same as Table A.3 for $\mathrm{Ca}$ and Ti.

\begin{tabular}{|c|c|c|c|c|c|c|c|c|}
\hline Star & $A(\mathrm{CaI})$ & $N$ lines & $A(\mathrm{CaII})$ & $N$ lines & $A(\mathrm{TiI})$ & $N$ lines & $A(\mathrm{TiII})$ & $N$ lines \\
\hline HD 20670 & $6.55 \pm 0.18$ & 4 & $6.55 \pm 0.08$ & 4 & $5.07 \pm 0.07$ & 5 & 4.90 & 1 \\
\hline HD 24040 & $6.49 \pm 0.12$ & 3 & $6.52 \pm 0.06$ & 4 & $5.09 \pm 0.10$ & 5 & 4.88 & 1 \\
\hline HD 28005 & $6.60 \pm 0.09$ & 3 & $6.62 \pm 0.07$ & 3 & $5.18 \pm 0.10$ & 5 & 4.99 & 1 \\
\hline HD 32673 & $6.47 \pm 0.14$ & 3 & $6.47 \pm 0.16$ & 4 & $5.05 \pm 0.19$ & 6 & & 0 \\
\hline HD 34445 & $6.43 \pm 0.17$ & 4 & $6.48 \pm 0.07$ & 4 & $4.98 \pm 0.09$ & 4 & 4.84 & 1 \\
\hline HD 34575 & $6.63 \pm 0.05$ & 3 & $6.60 \pm 0.09$ & 4 & $5.13 \pm 0.22$ & 6 & 5.05 & 1 \\
\hline HD 44420 & $6.60 \pm 0.05$ & 3 & $6.75 \pm 0.05$ & 3 & $5.19 \pm 0.06$ & 5 & 5.01 & 1 \\
\hline HD 56303 & $6.53 \pm 0.14$ & 4 & $6.57 \pm 0.14$ & 4 & $4.99 \pm 0.05$ & 5 & 4.94 & 1 \\
\hline HD 67346 & $6.56 \pm 0.15$ & 3 & $6.71 \pm 0.09$ & 4 & $5.10 \pm 0.07$ & 5 & 5.03 & 1 \\
\hline HD 69056 & $6.50 \pm 0.10$ & 3 & $6.51 \pm 0.08$ & 4 & $5.07 \pm 0.20$ & 6 & 4.97 & 1 \\
\hline HD 69809 & $6.59 \pm 0.07$ & 3 & $6.61 \pm 0.11$ & 4 & $5.15 \pm 0.04$ & 5 & 5.07 & 1 \\
\hline HD 69960 & $6.62 \pm 0.04$ & 3 & $6.59 \pm 0.12$ & 3 & $5.15 \pm 0.24$ & 6 & 4.98 & 1 \\
\hline HD 73226 & $6.54 \pm 0.10$ & 3 & $6.61 \pm 0.14$ & 4 & $5.10 \pm 0.17$ & 4 & 4.99 & 1 \\
\hline HD 73933 & $6.54 \pm 0.15$ & 4 & $6.60 \pm 0.09$ & 3 & $5.07 \pm 0.14$ & 4 & 5.00 & 1 \\
\hline HD 76909 & $6.60 \pm 0.10$ & 3 & $6.67 \pm 0.01$ & 3 & $5.24 \pm 0.06$ & 5 & 5.05 & 1 \\
\hline HD 77519 & $6.63 \pm 0.20$ & 4 & $6.76 \pm 0.07$ & 4 & $5.11 \pm 0.17$ & 4 & 4.99 & 1 \\
\hline HD 82943 & $6.54 \pm 0.07$ & 3 & $6.65 \pm 0.11$ & 4 & $5.10 \pm 0.06$ & 5 & 4.98 & 1 \\
\hline HD 85301 & $6.55 \pm 0.01$ & 3 & $6.55 \pm 0.16$ & 4 & $5.02 \pm 0.13$ & 5 & 5.01 & 1 \\
\hline HD 87359 & $6.43 \pm 0.07$ & 3 & $6.42 \pm 0.09$ & 4 & $4.89 \pm 0.19$ & 6 & 4.86 & 1 \\
\hline HD 87836 & $6.61 \pm 0.07$ & 3 & $6.64 \pm 0.16$ & 3 & $5.07 \pm 0.21$ & 5 & 4.95 & 1 \\
\hline HD 90681 & $6.61 \pm 0.12$ & 3 & $6.66 \pm 0.03$ & 3 & $5.14 \pm 0.10$ & 4 & 5.05 & 1 \\
\hline HD 90722 & $6.62 \pm 0.10$ & 3 & $6.67 \pm 0.14$ & 3 & $5.15 \pm 0.23$ & 5 & 5.18 & 1 \\
\hline HD 92788 & $6.60 \pm 0.07$ & 3 & $6.78 \pm 0.38$ & 4 & $5.06 \pm 0.21$ & 6 & 5.08 & 1 \\
\hline HD 97645 & $6.69 \pm 0.11$ & 4 & $6.62 \pm 0.10$ & 4 & $5.16 \pm 0.10$ & 3 & 5.09 & 1 \\
\hline HD 98618 & $6.35 \pm 0.09$ & 3 & $6.41 \pm 0.11$ & 4 & $4.78 \pm 0.14$ & 5 & 4.82 & 1 \\
\hline HD 98736 & $6.68 \pm 0.10$ & 3 & $6.73 \pm 0.08$ & 3 & $5.24 \pm 0.23$ & 4 & 5.05 & 1 \\
\hline HD 99491 & $6.70 \pm 0.09$ & 3 & $6.53 \pm 0.20$ & 2 & $5.27 \pm 0.19$ & 6 & 5.12 & 1 \\
\hline HD 99492 & $6.74 \pm 0.05$ & 3 & $6.74 \pm 0.49$ & 5 & 5.20 & 1 & 5.11 & 1 \\
\hline HD 100069 & $6.43 \pm 0.13$ & 4 & $6.46 \pm 0.13$ & 3 & 5.06 & 1 & & 0 \\
\hline HD 105631 & $6.50 \pm 0.12$ & 3 & $6.44 \pm 0.04$ & 3 & $5.01 \pm 0.22$ & 5 & 4.78 & 1 \\
\hline HD 106116 & $6.52 \pm 0.04$ & 3 & $6.50 \pm 0.10$ & 4 & $4.94 \pm 0.25$ & 5 & 4.88 & 1 \\
\hline HD 106156 & $6.59 \pm 0.08$ & 3 & $6.47 \pm 0.25$ & 3 & $5.15 \pm 0.20$ & 6 & 5.03 & 1 \\
\hline HD 108942 & $6.60 \pm 0.08$ & 3 & $6.51 \pm 0.16$ & 4 & $5.19 \pm 0.08$ & 4 & 4.98 & 1 \\
\hline HD 114174 & $6.40 \pm 0.04$ & 3 & $6.48 \pm 0.11$ & 4 & $4.99 \pm 0.05$ & 4 & 4.86 & 1 \\
\hline HD 116321 & $6.52 \pm 0.11$ & 4 & $6.68 \pm 0.16$ & 4 & $5.26 \pm 0.27$ & 3 & 5.33 & 1 \\
\hline HD 136618 & $6.55 \pm 0.15$ & 3 & $6.58 \pm 0.11$ & 3 & $5.10 \pm 0.08$ & 5 & 5.01 & 1 \\
\hline HD 145675 & $6.73 \pm 0.09$ & 3 & $6.83 \pm 0.06$ & 3 & $5.37 \pm 0.20$ & 5 & & 0 \\
\hline HD 147231 & $6.45 \pm 0.04$ & 3 & $6.34 \pm 0.18$ & 5 & $4.92 \pm 0.21$ & 5 & 4.82 & 1 \\
\hline HD 159222 & $6.41 \pm 0.10$ & 3 & $6.62 \pm 0.05$ & 4 & $4.94 \pm 0.02$ & 3 & 4.89 & 1 \\
\hline HD 190360 & $6.59 \pm 0.10$ & 3 & $6.71 \pm 0.21$ & 4 & $5.01 \pm 0.27$ & 5 & 5.08 & 1 \\
\hline
\end{tabular}


Table A.5. Same as Table A.2 for Cr, Ni, and Sr.

\begin{tabular}{|c|c|c|c|c|c|c|}
\hline Star & $A(\mathrm{Cr})$ & $N$ lines & $A(\mathrm{Ni})$ & $N$ lines & $A(\mathrm{Sr})$ & $N$ lines \\
\hline HD 20670 & $5.67 \pm 0.13$ & 8 & $6.48 \pm 0.17$ & 8 & 3.40 & 1 \\
\hline HD 24040 & $5.65 \pm 0.13$ & 9 & $6.50 \pm 0.16$ & 9 & 3.29 & 1 \\
\hline HD 28005 & $5.79 \pm 0.09$ & 8 & $6.64 \pm 0.19$ & 8 & 3.39 & 1 \\
\hline HD 32673 & $5.64 \pm 0.14$ & 8 & $6.46 \pm 0.18$ & 9 & 3.34 & 1 \\
\hline HD 34445 & $5.57 \pm 0.11$ & 9 & $6.35 \pm 0.21$ & 7 & & 0 \\
\hline HD 34575 & $5.78 \pm 0.10$ & 8 & $6.59 \pm 0.20$ & 9 & 3.20 & 1 \\
\hline HD 44420 & $5.76 \pm 0.13$ & 8 & $6.64 \pm 0.19$ & 8 & 3.45 & 1 \\
\hline HD 56303 & $5.58 \pm 0.11$ & 8 & $6.36 \pm 0.20$ & 10 & 3.42 & 1 \\
\hline HD 67346 & $5.70 \pm 0.10$ & 6 & $6.54 \pm 0.26$ & 10 & 3.49 & 1 \\
\hline HD 69056 & $5.67 \pm 0.13$ & 9 & $6.42 \pm 0.20$ & 9 & $3.36 \pm 0.19$ & 3 \\
\hline HD 69809 & $5.79 \pm 0.14$ & 8 & $6.65 \pm 0.11$ & 7 & 3.27 & 1 \\
\hline HD 69960 & $5.80 \pm 0.09$ & 7 & $6.58 \pm 0.19$ & 9 & 3.29 & 1 \\
\hline HD 73226 & $5.65 \pm 0.15$ & 9 & $6.37 \pm 0.21$ & 8 & 3.14 & 1 \\
\hline HD 73933 & $5.71 \pm 0.14$ & 4 & $6.31 \pm 0.22$ & 8 & 3.34 & 1 \\
\hline HD 76909 & $5.81 \pm 0.15$ & 7 & $6.64 \pm 0.22$ & 9 & 3.26 & 1 \\
\hline HD 77519 & $5.70 \pm 0.13$ & 6 & $6.49 \pm 0.19$ & 8 & 3.54 & 1 \\
\hline HD 82943 & $5.74 \pm 0.13$ & 8 & $6.49 \pm 0.16$ & 9 & 3.42 & 1 \\
\hline HD 85301 & $5.67 \pm 0.14$ & 9 & $6.32 \pm 0.25$ & 8 & & 0 \\
\hline HD 87359 & $5.57 \pm 0.13$ & 9 & $6.23 \pm 0.21$ & 9 & $3.35 \pm 0.27$ & 2 \\
\hline HD 87836 & $5.75 \pm 0.11$ & 7 & $6.63 \pm 0.27$ & 8 & 3.23 & 1 \\
\hline HD 90681 & $5.75 \pm 0.13$ & 8 & $6.52 \pm 0.21$ & 8 & 3.33 & 1 \\
\hline HD 90722 & $5.80 \pm 0.13$ & 9 & $6.65 \pm 0.22$ & 6 & & 0 \\
\hline HD 92788 & $5.77 \pm 0.14$ & 9 & $6.60 \pm 0.15$ & 8 & 3.17 & 1 \\
\hline HD 97645 & $5.72 \pm 0.19$ & 3 & $6.53 \pm 0.22$ & 9 & 3.52 & 1 \\
\hline HD 98618 & $5.50 \pm 0.13$ & 7 & $6.20 \pm 0.18$ & 8 & $3.29 \pm 0.18$ & 2 \\
\hline HD 98736 & $5.86 \pm 0.08$ & 6 & $6.79 \pm 0.26$ & 8 & $3.45 \pm 0.03$ & 2 \\
\hline HD 99491 & $5.91 \pm 0.14$ & 9 & $6.63 \pm 0.20$ & 8 & $3.44 \pm 0.18$ & 2 \\
\hline HD 99492 & $5.93 \pm 0.07$ & 7 & $6.70 \pm 0.18$ & 7 & $3.53 \pm 0.04$ & 2 \\
\hline HD 100069 & $5.52 \pm 0.09$ & 8 & $6.35 \pm 0.19$ & 8 & 3.22 & 1 \\
\hline HD 105631 & $5.69 \pm 0.11$ & 9 & $6.39 \pm 0.27$ & 10 & 3.17 & 1 \\
\hline HD 106116 & $5.63 \pm 0.13$ & 8 & $6.39 \pm 0.26$ & 9 & 3.22 & 1 \\
\hline HD 106156 & $5.74 \pm 0.08$ & 8 & $6.50 \pm 0.25$ & 9 & 3.18 & 1 \\
\hline HD 108942 & $5.79 \pm 0.13$ & 7 & $6.62 \pm 0.21$ & 9 & 3.42 & 1 \\
\hline HD 114174 & $5.55 \pm 0.09$ & 7 & $6.19 \pm 0.20$ & 8 & & 0 \\
\hline HD 116321 & $5.73 \pm 0.19$ & 3 & $6.49 \pm 0.18$ & 9 & 3.51 & 1 \\
\hline HD 136618 & $5.71 \pm 0.13$ & 7 & $6.56 \pm 0.20$ & 9 & 3.47 & 1 \\
\hline HD 145675 & $5.95 \pm 0.13$ & 8 & $6.75 \pm 0.28$ & 8 & & 0 \\
\hline HD 147231 & $5.50 \pm 0.10$ & 8 & $6.29 \pm 0.17$ & 8 & $3.23 \pm 0.13$ & 3 \\
\hline HD 159222 & $5.58 \pm 0.13$ & 8 & $6.41 \pm 0.22$ & 8 & 3.23 & 1 \\
\hline HD 190360 & $5.66 \pm 0.15$ & 9 & $6.39 \pm 0.24$ & 8 & 3.47 & 1 \\
\hline
\end{tabular}

